\title{
Random Function Iterations for Consistent Stochastic Feasibility
}

\author{
Neal Hermer, D. Russell Luke ${ }^{\dagger}$ and Anja Sturm ${ }^{\ddagger}$
}

September 19, 2018

\begin{abstract}
We study the convergence of iterated random functions for stochastic feasibility in the consistent case (in the sense of Butnariu and Flåm [1]) in several different settings, under decreasingly restrictive regularity assumptions of the fixed point mappings. The iterations are Markov chains and, for the purposes of this study, convergence is understood in very restrictive terms. We show that sufficient conditions for geometric (linear) convergence in expectation of stochastic projection algorithms presented in Nedić [2], are in fact necessary for geometric (linear) convergence in expectation more generally of iterated random functions.
\end{abstract}

2010 Mathematics Subject Classification: Primary 60J05, 52A22, 49J55 Secondary 49J53, 65K05.

Keywords: Averaged mappings, nonexpansive mappings, paracontractions, stochastic feasibility, stochastic fixed point problem, iterated random functions, metric subregularity, linear regularity, linear convergence in expectation, geometric convergence of measures

\section{Introduction}

We are inspired by problems of the form

$$
\text { Find } x^{*} \in \bigcap_{i \in I} C_{i} \text {. }
$$

where $I$ is an index set and $C_{i}$ are closed subsets of a metric space. When $I$ is a finite set, then this is the classical deterministic feasibility problem. Randomized algorithms for solving such finite intersections have been intensely studied in recent years for their application to distributed computational schemes and machine learning. Our study here concerns a generalization where $I$ is an arbitrary (possibly uncountable) set. This was first considered by Butnariu and Flåm

*Institut für Numerische und Angewandte Mathematik, Universität Göttingen, 37083 Göttingen, Germany. NH was supported by Deutsche Forschungsgemeinschaft Research Training Grant 2088 TP-B5. E-mail: n.hermer@math. uni-goettingen.de

${ }^{\dagger}$ Institut für Numerische und Angewandte Mathematik, Universität Göttingen, 37083 Göttingen, Germany. DRL was supported in part by Deutsche Forschungsgemeinschaft Research Training Grant 2088 TP-B5. Email: r.luke@math.uni-goettingen.de

${ }^{\ddagger}$ Institut für Mathematische Stochastik, Universität Göttingen, 37077 Göttingen, Germany. AS was supported in part by Deutsche Forschungsgemeinschaft Research Training Grant 2088 TP-B5. E-mail: asturm@math.uni-goettingen.de 
$[1,3,4]$, where this is called the stochastic feasibility problem. There are many reasons why one might consider such infinite feasibility problems. At the time Butnariu and Flåm's work appeared, there was a great deal of interest in solution methods for linear integral equations. Our primary motivation is to propose a new way of modeling and analyzing errors, either numerical or measurement, as they are manifest in numerical iterative procedures.

The simplest algorithm one could imagine for solving such problems is to generate sequences $\left(x_{k}\right)_{k \in \mathbb{N}}$ by the fixed point iteration

$$
x_{k+1} \in\left(\prod_{i \in I} P_{C_{i}}\right) x_{k}
$$

where $P_{C_{i}}$ is the metric projection onto the set $C_{i}$ and $\prod_{i \in I}$ indicates the composition of the operators over the index set. This is known as the cyclic projections algorithm and has been extensively studied for the case when $I$ is a finite set. But when $I$ is infinite one immediately encounters the problem that such an algorithm never completes the first iteration!

One application where infinite feasibility problems appear very naturally is integral equations of the first kind in the separable Hilbert space $L_{2}([a, b])$, as considered in [4]:

$$
(T x)(t)=\int_{a}^{b} K(t, s) x(s) \mathrm{d} s=g(t) \quad t \in[a, b],
$$

with $g \in L_{2}([a, b])$. The feasibility reformulation of this problem is

$$
\text { Find } x \in \bigcap_{t \in[a, b], \text { a.s. }} C_{t}:=\left\{\varphi \in L_{2}([a, b]) \mid(T \varphi)(t)=g(t)\right\} .
$$

The almost surely (a.s) under the intersection will be clarified below. The basic idea, however, is to choose the parameter $t$ above randomly and to choose the nearest point in the set $C_{t}$ to the current guess. As such, the sequences that we generate are random processes. We return to this application in Section 4.3.

One of our main contributions is to place the previous results on stochastic projections iterations in the broader context of random function iterations, that is, iterations of randomly selected mappings with arbitrary initial distributions from which the initial points are chosen. Following [5] we characterize the sequence generated by the method of Random Function Iterations (Algorithm 1) as a Markov chain (Proposition 2.2). There are many different notions of convergence of Markov chains. For consistent feasibility problems considered in this study (Standing Assumption 2) almost sure convergence applies, which perhaps is of limited interest in the broader context of generic Markov chains. When we move to inconsistent stochastic feasibility problems, what we formulate more generally as stochastic fixed point problems in a follow-up study, the richness of the theory of Markov processes comes much more into play. For the consistent case we are able to establish convergence results in a number of new settings, namely compact metric spaces or $\mathbb{R}^{n}$ with paracontracting mappings (see Section 3.1 and 3.2 , resp.) and separable Hilbert spaces with averaged mappings (see Section 3.3). (It is the technology of averaged mappings that opens the door to an analysis of algorithms for inconsistent stochastic feasibility problems and more generally stochastic fixed point problems.)

Our framework allows us to address a variety of Markov chains and stochastic algorithms, though to fix these ideas our main example will be stochastic sequential projection algorithms. We also achieve with this analysis more refined convergence statements about the corresponding sequences of random variables, namely almost surely strong and, under certain assumptions, geometric convergence (called linear or exponential in other communities) of the measures Theorem 3.12. We then show necessary and sufficient conditions for geometric convergence in expectation Theorem 3.15 in a stochastic analog to [6, Theorem 3.12 and Corollary 3.13]. These 
conditions are identified as a manifestation of metric subregularity of a suitable merit function at its zeros. When specialized to convex feasibility, this formulation of metric subregularity of the merit function is equivalent to the function possessing what is known as the KL property Proposition 4.2. Finally, we identify a previously unrecognized necessary condition for geometric convergence of stochastic sequential projection algorithms, Theorem 4.3. Despite the strong assumptions of the present study, a number of unexpected behaviors can occur; these are demonstrated in concrete examples.

\section{Stochastic Fixed Point Theory}

Consider a collection of continuous mappings $T_{i}: G \rightarrow G, i \in I$, on a metric space $(G, d)$, where $I$ is an arbitrary index set. Assume that $(I, \mathcal{I})$ is a measurable space. Let $(\Omega, \mathcal{F}, \mathbb{P})$ be a probability space. Let $\xi$ be an $I$-valued random variable, i.e. $\xi: \Omega \rightarrow I$ measurable $\left(\xi^{-1} A \in \mathcal{F}\right.$ for all $A \in \mathcal{I})$.

Let $\left(\xi_{n}\right)_{n \in \mathbb{N}}$ be an iid sequence with $\xi_{n} \stackrel{\mathrm{d}}{=} \xi$. Let $\mu$ be a probability measure on $(G, \mathcal{B}(G))$. The stochastic selection method is given by

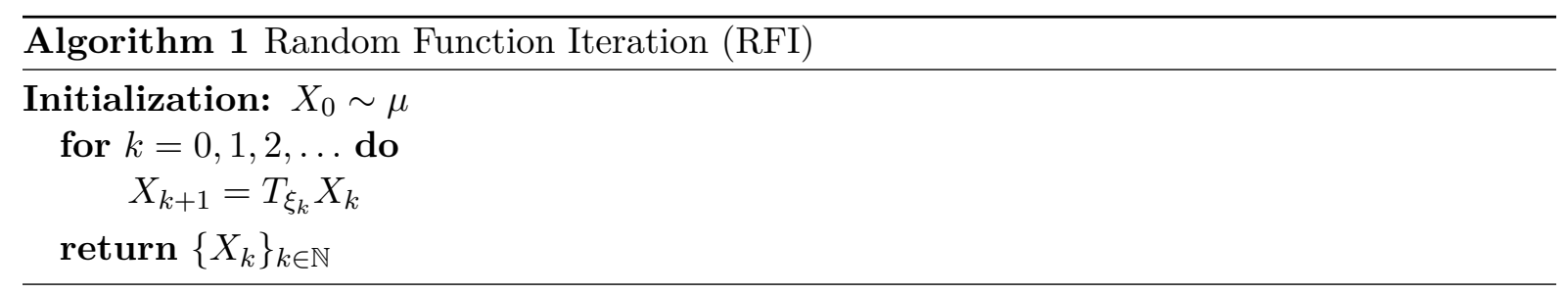

Here we mean by $X_{0} \sim \mu$, that the law (i.e. distribution) of $X_{0}$ satisfies $\mathcal{L}\left(X_{0}\right):=\mathbb{P}^{X_{0}}:=$ $\mathbb{P}\left(X_{0} \in \cdot\right):=\mathbb{P} \circ X_{0}^{-1}=\mu$. The following assumptions will be employed throughout.

\section{Standing Assumption 1}

a) $X_{0}, \xi_{0}, \xi_{1}, \ldots, \xi_{k}$ are independent for every $k \in \mathbb{N}_{0}$, where $\left(\xi_{k}\right)_{k \in \mathbb{N}}$ are i.i.d.

b) The function $\Phi: G \times I \rightarrow G,(x, i) \mapsto T_{i} x$ is measurable.

\subsection{RFI as a Markov chain}

The iterates of the RFI $X_{k+1}=\Phi\left(X_{k}, \xi_{k}\right)=T_{\xi_{k}} X_{k}, k \in \mathbb{N}_{0}$, can be considered as a time homogeneous Markov chain with transition kernel

$$
(x \in G)(A \in \mathcal{B}(G)) \quad p(x, A):=\mathbb{P}(\Phi(x, \xi) \in A)=\mathbb{P}\left(T_{\xi} x \in A\right)
$$

where $\Phi$ is called an update function.

To see that $p$ is really a transition kernel, recall that, in general, a transition kernel $p$ : $G \times \mathcal{B}(G) \rightarrow[0,1]$ is measurable in the first argument, i.e. $p(\cdot, A)$ is measurable for all $A \in \mathcal{B}(G)$ (follows from [7, Lemma 1.26]) and is a probability measure in the second argument, i.e. $p(x, \cdot)$ is a probability measure for all $x \in G$ (immediate by definition). Now recall the definition of a Markov chain with general transition kernel $p$.

Definition 2.1. A sequence of random variables $\left(X_{k}\right)_{k \in \mathbb{N}_{0}}, X_{k}:(\Omega, \mathcal{F}, \mathbb{P}) \rightarrow(G, \mathcal{B}(G))$ is called Markov chain with transition kernel $p$ if for all $k \in \mathbb{N}_{0}$ and $A \in \mathcal{B}(G) \mathbb{P}$-a.s. holds

(i) $\mathbb{P}\left(X_{k+1} \in A \mid X_{0}, X_{1}, \ldots, X_{k}\right)=\mathbb{P}\left(X_{k+1} \in A \mid X_{k}\right)$ 
(ii) $\mathbb{P}\left(X_{k+1} \in A \mid X_{k}\right)=p\left(X_{k}, A\right)$.

Since $(G, \mathcal{B}(G))$ is a Borel space and $X_{k}$ is a random variable in $G$, it makes sense to talk about these conditional expectations (existence of regular versions of the conditional distribution by [7, Theorem 5.3]).

From the setting above, the following fact follows easily from Theorem A.9.

Proposition 2.2. Under Standing Assumption 1, the sequence of random variables $\left(X_{k}\right)_{k \in \mathbb{N}_{0}}$ generated by Algorithm 1 is a Markov chain with transition kernel p given by (3).

Let $\mu \in \mathscr{P}(G)$, where $\mathscr{P}(G)$ is the space of all probability measures on $G$. The Markov operator $\mathcal{P}$ acting on a measure $\mu$ is defined via

$$
(A \in \mathcal{B}(G)) \quad \mu \mathcal{P}(A):=\int_{G} p(x, A) \mu(\mathrm{d} x) .
$$

One defines the operation of the Markov operator acting on a measurable function $f: G \rightarrow \mathbb{R}$ via

$$
(x \in G) \quad \mathcal{P} f(x):=\int_{G} f(y) p(x, \mathrm{~d} y) .
$$

Note that

$$
\mathcal{P} f(x)=\int_{G} f(y) \mathbb{P}^{\Phi(x, \xi)}(\mathrm{d} y)=\int_{\Omega} f(\Phi(x, \xi(\omega))) \mathbb{P}(\mathrm{d} \omega)=\int_{I} f(\Phi(x, u)) \mathbb{P}^{\xi}(\mathrm{d} u) .
$$

There are several notions of convergence that one can study for the sequence $\left(X_{k}\right)$ on the metric space $(G, d)$, or correspondingly of the law $\left(\mathcal{L}\left(X_{k}\right)\right)$ on $\mathscr{P}(G)$, where $\mathcal{L}\left(X_{k}\right):=\mathbb{P}^{X_{k}}:=$ $\mathbb{P}\left(X_{k} \in \cdot\right)$. Denote the set of bounded and continuous functions from $G$ to $\mathbb{R}$ by $C_{b}(G)$. Let $\left(\nu_{n}\right)$ be a sequence of probability measures on $G$. The sequence $\left(\nu_{n}\right)$ converges to $\nu$ in the weak sense, if $\nu \in \mathscr{P}(G)$ and for all $f \in C_{b}(G)$ it holds that $\nu_{n} f \rightarrow \nu f$ as $n \rightarrow \infty$.

A fixed point of the Markov operator $\mathcal{P}$ is called an invariant distribution, i.e. $\pi \in \mathscr{P}(G)$ is invariant if and only if $\pi \mathcal{P}=\pi$. A very general notion of convergence in the context of Markov chains concerns the convergence of the probability measures $\nu_{k}:=\frac{1}{k} \sum_{j=1}^{k} \mathcal{L}\left(X_{j}\right)$ in the weak sense. An elementary fact from the theory of Markov chains (Theorem A.4) is that, if this converges, it converges to an invariant probability measure $\pi$ : For $f \in C_{b}(G)$,

$$
\nu_{k} f=\mathbb{E}\left[\frac{1}{k} \sum_{j=1}^{k} f\left(X_{j}\right)\right] \rightarrow \pi f, \quad \text { as } k \rightarrow \infty .
$$

The notion of convergence we consider here is much stronger; we consider almost sure convergence of the sequence $\left(X_{k}\right)$ to a random variable $X$ :

$$
X_{k} \rightarrow X \text { a.s., } \quad \text { as } k \rightarrow \infty \text {. }
$$

Clearly, almost sure convergence of the sequence implies the more general notion above. This is common in the studies of stochastic algorithms in optimization, though this does not require the full power of the theory of general Markov processes. For consistent feasibility (defined below) however, this is all that is needed for our present purposes. In a follow-up study we will need to consider more general notions of convergence of this Markov chain. 


\subsection{Consistent Stochastic Feasibility Problem}

The stochastic feasibility problem is to find a point

$$
x^{*} \in C:=\left\{x \in G \mid \mathbb{P}\left(x \in \operatorname{Fix} T_{\xi}\right)=1\right\},
$$

where the fixed point set of the operator $T_{i}$ is denoted as

$$
\text { Fix } T_{i}=\left\{x \in G \mid x=T_{i} x\right\} .
$$

We assume throughout that not only is $\operatorname{Fix} T_{i}$ non-empty for $\mathbb{P}^{\xi}$-almost all $i \in I$, but more restrictively:

Standing Assumption 2 (consistent feasibility problem). The set $C$ is nonempty.

Note that due to continuity of $T_{i}$ it follows, that Fix $T_{i}$ is a closed set. This specializes immediately to the stochastic feasibility problem formulated by Butnariu and Flåm [1] where Fix $T_{\xi}=C_{\xi}$. In order to make sense of the specialization to stochastic set feasibility, we need the event $\left\{x \in \operatorname{Fix} T_{\xi}\right\}$ to be an element of $\mathcal{F}$ for any $x \in G$.

Remark 2.3: Since $\{x\} \in \mathcal{B}(G)$ and the function $\Phi_{\xi}: G \times \Omega \rightarrow G,(x, \omega) \mapsto \Phi_{\xi}(x, \omega):=$ $(\Phi \circ(\operatorname{Id}, \xi))(x, \omega)=T_{\xi(\omega)} x$ is measurable as composition of two measurable functions, we find

$$
\begin{aligned}
\left\{x \in \operatorname{Fix} T_{\xi}\right\} & =\left\{\omega \in \Omega \mid x \in \operatorname{Fix} T_{\xi(\omega)}\right\} \\
& =\left\{\omega \in \Omega \mid T_{\xi(\omega)} x=x\right\} \\
& =\left\{\omega \in \Omega \mid(x, \omega) \in \Phi_{\xi}^{-1}\{x\}\right\} \\
& \in \mathcal{F},
\end{aligned}
$$

since slices of sets in the product $\sigma$-field are measurable with respect to the single $\sigma$-fields (see Lemma A.1).

Denote in the following for $A \subset \Omega, C(A):=\bigcap_{\omega \in A} \operatorname{Fix} T_{\xi(\omega)}$.

Lemma 2.4 (equivalence of stochastic and deterministic feasibility problems). Under the standing assumptions, and if $G$ is complete and separable, there exists a $\mathbb{P}$-nullset $N \subset \Omega$, such that

$$
C=C(\Omega \backslash N)=\bigcap_{\omega \in \Omega \backslash N} \operatorname{Fix} T_{\xi(\omega)} .
$$

Furthermore, $C \subset G$ is closed.

Proof. For the direction " $\supset$ ", note that $\mathbb{P}(\Omega \backslash N)=1$ for any $\mathbb{P}$-nullset $N \subset \Omega$, so for $x \in C(\Omega \backslash N)$ it holds that $\mathbb{P}\left(x \in\right.$ Fix $\left.T_{\xi}\right)=1$, i.e. $x \in C$.

Consider now the direction " $\subset$ ". Let $Q$ be a dense and countable subset of $C$ (exists by Theorem A.2). Since for each $q \in Q, \mathbb{P}\left(q \in \operatorname{Fix} T_{\xi}\right)=1$, there is $N_{q} \subset \Omega$ with $\mathbb{P}\left(N_{q}\right)=0$ and $q \in C\left(\Omega \backslash N_{q}\right)$. Set $N=\bigcup_{q \in Q} N_{q}$, then $\mathbb{P}(N)=0$ and $q \in C(\Omega \backslash N)$ for all $q \in Q$.

Now let $c \in C$, so $\exists\left(q_{n}\right)_{n \in \mathbb{N}} \subset Q$ with $q_{n} \rightarrow c$ as $n \rightarrow \infty$. Since, for all $i \in I$, Fix $T_{i}$ is closed by continuity of $T_{i}$, we get $c=\lim _{n \rightarrow \infty} q_{n} \in C(\Omega \backslash N)$.

The set $C(\Omega \backslash N)$ is defined as intersection over closed sets and hence closed itself.

Remark 2.5 (interpretation): Lemma 2.4 shows that the feasible set $C$ in the separable case can be written as intersection of a selection of sets $\operatorname{Fix} T_{\xi(\omega)}$ as in the deterministic formulation of the fixed point problem, but where $\omega \in \Omega \backslash N$ for a nullset $N \subset \Omega$. In fact $C(\Omega)$ is in general a proper subset of $C=C(\Omega \backslash N)$ or can even be empty. But note that, even though 
the construction of $C$ in Lemma 2.4 appears to depend on the random variable $\xi$, in fact $C$ only depends on the distribution $\mathbb{P}^{\xi}$ by definition. Furthermore, in the context of more general Markov chains, we have,

$$
(c \in C) \quad p(c,\{c\})=\mathbb{P}\left(T_{\xi} c \in\{c\}\right)=\mathbb{P}(\Omega \backslash N)=1 .
$$

Hence

$$
(A \in \mathcal{B}(G)) \quad \delta_{c} \mathcal{P}(A)=p(c, A)=\mathbb{1}_{A}(c)=\delta_{c}(A) .
$$

In other words, the delta function $\delta_{c}$ for $c \in C$ is an invariant measure for $\mathcal{P}$.

Corollary 2.6 ( $\mathbb{P}^{\xi}$ nullset, separable space). Under the assumptions of Lemma 2.4 there exists a $\mathbb{P}$-nullset $N$ with $C=C(\Omega \backslash N)$, such that $\xi(N):=\{\xi(\omega) \mid \omega \in N\}$ is a $\mathbb{P}^{\xi}$-nullset, where we denote $\mathbb{P}^{\xi}=\mathbb{P}(\xi \in \cdot)$, and it satisfies

$$
C=\bigcap_{i \in \xi(\Omega) \backslash \xi(N)} \operatorname{Fix} T_{i}
$$

Proof. We will construct a $\mathbb{P}$-nullset $N$ for which $\xi(\Omega \backslash N)=\xi(\Omega) \backslash \xi(N)$, where $\xi(N)$ is a $\mathbb{P}^{\xi}$-nullset, in that case immediately follows that

$$
\bigcap_{\omega \in \Omega \backslash N} \operatorname{Fix} T_{\xi(\omega)}=\bigcap_{i \in \xi(\Omega) \backslash \xi(N)} \operatorname{Fix} T_{i} .
$$

Let $A_{x}:=\left\{i \in I \mid T_{i} x=x\right\}$ for $x \in G$, then analogously to Remark 2.3

$$
A_{x}=\left\{i \in I \mid(x, i) \in \Phi^{-1}\{x\}\right\} \in \mathcal{I}
$$

and so is $A:=\bigcap_{c \in C} A_{c}=\bigcap_{q \in Q} A_{q}$ as countable intersection of measurable sets $(Q \subset C$ dense and countable, see proof of Lemma 2.4). Let $\tilde{N}$ be the $\mathbb{P}$-nullset from Lemma 2.4, i.e. $C=C(\Omega \backslash \tilde{N})$, note that due to

$$
C=\bigcap_{\omega \in \Omega \backslash \tilde{N}} \operatorname{Fix} T_{\xi(\omega)}=\bigcap_{i \in \xi(\Omega \backslash \tilde{N})} \operatorname{Fix} T_{i} \neq \emptyset
$$

it holds $\xi(\Omega \backslash \tilde{N}) \subset A_{c} \neq \emptyset$, for all $c \in C$. Set $N:=\Omega \backslash \xi^{-1} A$, then from $\Omega \backslash \tilde{N} \subset \xi^{-1} A$ follows $N \subset \tilde{N}$ is a $\mathbb{P}$-nullset and

$$
\mathbb{P}^{\xi}(A)=\mathbb{P}\left(\xi^{-1} A\right) \geq \mathbb{P}(\Omega \backslash \tilde{N})=1,
$$

i.e. $\mathbb{P}^{\xi}(\xi(N))=1-\mathbb{P}^{\xi}(A)=0$. By definition of $A$ we have for $\omega \in \xi^{-1} A$, that any $c \in C$ satisfies $c \in \operatorname{Fix} T_{\xi(\omega)}$, so it follows $C \subset C\left(\xi^{-1} A\right)$. Due to $C(\Omega \backslash N) \subset C(\Omega \backslash \tilde{N})$ holds

$$
C=\bigcap_{\omega \in \xi^{-1} A} \operatorname{Fix} T_{\xi(\omega)}=\bigcap_{i \in \xi\left(\xi^{-1} A\right)} \operatorname{Fix} T_{i}=\bigcap_{i \in \xi(\Omega) \backslash \xi(N)} \operatorname{Fix} T_{i} .
$$

Note that from $N=\Omega \backslash \xi^{-1} A$ follows $\xi(N)=\xi(\Omega) \backslash \xi\left(\xi^{-1} A\right)$.

If $\xi$ is not surjective, then $\xi(\Omega) \neq I$. In that case, there is a $\mathbb{P}^{\xi}$-nullset $\xi(N)$ of indices in $I$, that are not needed to characterize the fixed point set, and these indices can be removed from the index set $I$. Note also that, in general, the $\mathbb{P}$-nullsets occurring in Lemma 2.4 and Corollary 2.6 are different. If there is $N \subset \Omega$ with $C=C(\Omega \backslash N)$, then it need not be the case that $C=\bigcap_{i \in \xi(\Omega) \backslash \xi(N)} \operatorname{Fix} T_{i}$.

In the context of the iterates $X_{k}$ of Algorithm 1 in many of the results below we construct the set $N$ in Lemma 2.4 as follows:

$$
N=\bigcup_{k} N_{k} \quad \text { where } N_{k}:=\Omega \backslash\left\{\omega \in \Omega \mid T_{\xi_{k}(\omega)} c=c \forall c \in C\right\} .
$$

From Lemma 2.4 we have that $N_{k}$ is a set of measure zero, hence so is $N$. 


\section{Convergence Analysis}

We achieve convergence of iterated random functions for consistent stochastic feasibility in several different settings under different assumptions on the metric spaces and the mappings $T_{i}$ $(i \in I)$. The main properties of the mappings we consider are:

- quasi-nonexpansive mappings, i.e.

$$
\left(\forall x \notin \operatorname{Fix} T_{i}\right)\left(\forall y \in \operatorname{Fix} T_{i}\right) \quad d\left(T_{i} x, y\right) \leq d(x, y) .
$$

- paracontractions, i.e. $T_{i}$ is continuous and

$$
\left(\forall x \notin \operatorname{Fix} T_{i}\right)\left(\forall y \in \operatorname{Fix} T_{i}\right) \quad d\left(T_{i} x, y\right)<d(x, y) ;
$$

- nonexpansive mappings, i.e.

$$
(\forall x, y \in G) \quad d\left(T_{i} x, T_{i} y\right) \leq d(x, y)
$$

- averaged mappings on a normed linear space $\mathcal{H}$, i.e. mappings $T: \mathcal{H} \rightarrow \mathcal{H}$ for which there exists an $\alpha \in(0,1)$ such that

$$
(\forall x, y \in \mathcal{H}) \quad\|T x-T y\|^{2}+\frac{1-\alpha}{\alpha}\|(x-T x)-(y-T y)\|^{2} \leq\|x-y\|^{2} .
$$

Note that for a quasi-nonexpansive mapping $T: G \rightarrow G$ the condition $x \in$ Fix $T$ implies that $d(T x, y)=d(x, y)$ for all $y \in G$. The set of quasi-nonexpansive mappings contains the paracontractions and the nonexpansive mappings. The set of projectors onto convex sets or more generally the set of averaged mappings on a Hilbert space $\mathcal{H}$ is contained in both the set of nonexpansive mappings and the set of paracontractions [8, Remark 4.24 and 4.26]. For an example of a paracontraction that is not averaged see Example 3.1 and Appendix B. Averaged mappings were first used in the work of Mann, Krasnoselski, Edelstein, Gurin, Polyak and Raik who wrote seminal papers in the analysis of (firmly) nonexpansive and averaged mappings [9-12] although the terminology "averaged" wasn't coined until sometime later [13].

Example 3.1. Let $f: \mathbb{R} \rightarrow \mathbb{R}_{+}$be continuous. Let $f(0)=0$ and $|f(x)|<|x|$ for all $x \in \mathbb{R} \backslash\{0\}$, then $f$ is paracontractive. This includes also convex functions, e.g. Huber functions, which are not averaged in general (see Appendix B). For other examples on $\mathbb{R}^{n}$ also see Appendix B.

\subsection{RFI on a compact metric space}

In this section we establish convergence of the RFI on a compact metric space. The next example illustrates why nonexpansivity alone does not suffice to guarantee convergence to the intersection set $C$.

Example 3.2 (nonexpansive mappings, negative result). For non-expansive mappings in general, one cannot expect that the support of every invariant measure is contained in the feasible set $C$. Consider a rotation in positive direction in $\mathbb{R}^{2}$

$$
A=\left(\begin{array}{cc}
\cos (\varphi) & -\sin (\varphi) \\
\sin (\varphi) & \cos (\varphi)
\end{array}\right), \quad \varphi \in(0,2 \pi)
$$

and set $\xi=1$ and $I=\{1\}, T_{1}=A$. Then $C=\{0\}$ and, since $\|A\|=1, A$ is nonexpansive, but $\|A x\|=\|x\|$ for all $x \in \mathbb{R}^{2}$. So the (deterministic) iteration $X_{k+1}=A X_{k}$ will not converge to 0 , whenever $X_{0} \sim \delta_{x}, x \neq 0$. 
A sufficient requirement on the mappings $T_{i}$ to ensure convergence of Algorithm 1 is paracontractiveness. The next Lemma is the main ingredient for proving a.s. convergence of $\left(X_{k}\right)$ to a random point in $C$. The support of a probability measure $\nu \in \mathscr{P}(G)$ is the smallest closed set $S \subset G$, for which $\nu(G \backslash S)=0$ (see also Theorem A.3 for equivalent representations); we then write $S=\operatorname{supp} \nu$.

Lemma 3.3 (invariant measures for para-contractions). Under the standing assumptions and if $T_{i}(i \in I)$ is paracontracting on a compact metric space, then the set of invariant measures for $\mathcal{P}$ is $\{\pi \in \mathscr{P}(G) \mid \operatorname{supp} \pi \subset C\}$.

Proof. It is clear that $\pi \in \mathscr{P}(G)$ with supp $\pi \subset C$ is invariant, since $p(x,\{x\})=\mathbb{P}\left(T_{\xi} x \in\{x\}\right)=$ $\mathbb{P}\left(x \in \operatorname{Fix} T_{\xi}\right)=1$ for all $x \in C$ and hence $\pi \mathcal{P}(A)=\int_{C} p(x, A) \pi(\mathrm{d} x)=\pi(A)$ for all $A \in \mathcal{B}(G)$.

The other implication is not so immediate. Suppose supp $\pi \backslash C \neq \emptyset$ for some invariant measure $\pi$ of $\mathcal{P}$. Then due to compactness of $\operatorname{supp} \pi$ (as it is closed in $G$ ) we can find $s \in \operatorname{supp} \pi$ maximizing the continuous function $\operatorname{dist}(\cdot, C)$ on $G$. So $d_{\max }=\operatorname{dist}(s, C)>0$. We show that the probability mass around $s$ will be attracted to the feasible set $C$, implying that the invariant measure loses mass around $s$ in every step, which yields a contradiction.

Define the set of points being more than $d_{\max }-\epsilon$ away from $C$ :

$$
K(\epsilon):=\left\{x \in G \mid \operatorname{dist}(x, C)>d_{\max }-\epsilon\right\}, \quad \epsilon \in\left(0, d_{\max }\right) .
$$

This set is measurable, i.e. $K(\epsilon) \in \mathcal{B}(G)$, because it is open. Let $M(\epsilon)$ be the event in $\mathcal{F}$, where $T_{\xi} s$ is at least $\epsilon$ closer to $C$ than $s$, i.e.

$$
M(\epsilon):=\left\{\omega \in \Omega \mid \operatorname{dist}\left(T_{\xi(\omega)} s, C\right) \leq d_{\max }-\epsilon\right\} .
$$

There are two possibilities, either there is an $\epsilon \in\left(0, d_{\max }\right)$ with $\mathbb{P}(M(\epsilon))>0$ or no such $\epsilon$ exists. In the latter case we have $\operatorname{dist}\left(T_{\xi} s, C\right)=d_{\max }=\operatorname{dist}(s, C)$ a.s. by paracontractiveness of $T_{i}$. By compactness of $C$ there exists $c \in C$ such that $0<d_{\max }=d(s, c)$. Hence the probability of the set of $\omega \in \Omega$ such that $s \notin \operatorname{Fix} T_{\xi(\omega)}$ is positive and so is the probability that $\operatorname{dist}\left(T_{\xi(\omega)} s, C\right) \leq d\left(T_{\xi(\omega)} s, c\right)<d(s, c)$ - a contradiction.

So it must hold that there is an $\epsilon \in\left(0, d_{\max }\right)$ with $\mathbb{P}(M(\epsilon))>0$. In view of continuity of the mappings $T_{i}$ around $s, i \in I$, define

$$
A_{n}:=\left\{\omega \in M(\epsilon) \mid d\left(T_{\xi(\omega)} x, T_{\xi(\omega)} s\right) \leq \frac{\epsilon}{2} \quad \forall x \in \mathbb{B}\left(s, \frac{1}{n}\right)\right\} \quad(n \in \mathbb{N}) .
$$

It holds that $A_{n} \subset A_{n+1}$ and $\mathbb{P}\left(\bigcup_{n} A_{n}\right)=\mathbb{P}(M(\epsilon))$. So in particular there is an $m \in \mathbb{N}, m \geq 2 / \epsilon$ with $\mathbb{P}\left(A_{m}\right)>0$. For all $x \in \mathbb{B}\left(s, \frac{1}{m}\right)$ and all $\omega \in A_{m}$ we have

$$
\operatorname{dist}\left(T_{\xi(\omega)} x, C\right) \leq d\left(T_{\xi(\omega)} x, T_{\xi(\omega)} s\right)+\operatorname{dist}\left(T_{\xi(\omega)} s, C\right) \leq d_{\max }-\frac{\epsilon}{2},
$$

which means $T_{\xi(\omega)} x \in G \backslash K\left(\frac{\epsilon}{2}\right)$. Hence, in particular we conclude that

$$
p\left(x, K\left(\frac{\epsilon}{2}\right)\right)<1 \quad \forall x \in \mathbb{B}\left(s, \frac{1}{m}\right) .
$$

Since $p(x, K(\epsilon))=0$ for $x \in G$ with $\operatorname{dist}(x, C) \leq d_{\max }-\epsilon$ due to paracontractiveness, it holds by invariance of $\pi$ that

$$
\pi(K(\epsilon))=\int_{G} p(x, K(\epsilon)) \pi(\mathrm{d} x)=\int_{K(\epsilon)} p(x, K(\epsilon)) \pi(\mathrm{d} x) .
$$


It follows, then, that

$$
\begin{aligned}
\pi\left(K\left(\frac{\epsilon}{2}\right)\right) & =\int_{K\left(\frac{\epsilon}{2}\right)} p\left(x, K\left(\frac{\epsilon}{2}\right)\right) \pi(\mathrm{d} x) \\
& =\int_{\mathbb{B}\left(s, \frac{1}{m}\right)} p\left(x, K\left(\frac{\epsilon}{2}\right)\right) \pi(\mathrm{d} x)+\int_{K\left(\frac{\epsilon}{2}\right) \backslash \mathbb{B}\left(s, \frac{1}{m}\right)} p\left(x, K\left(\frac{\epsilon}{2}\right)\right) \pi(\mathrm{d} x) \\
& <\pi\left(\mathbb{B}\left(s, \frac{1}{m}\right)\right)+\pi\left(K\left(\frac{\epsilon}{2}\right) \backslash \mathbb{B}\left(s, \frac{1}{m}\right)\right)=\pi\left(K\left(\frac{\epsilon}{2}\right)\right)
\end{aligned}
$$

which is a contradiction. So the assumption that $\operatorname{supp} \pi \backslash C \neq \emptyset$ is false, i.e. $\operatorname{supp} \pi \subset C$ as claimed.

Theorem 3.4 (Theorem 4.22 in [14]). Under Standing Assumption 1, if $T_{i}(i \in I)$ is continuous, then the Markov operator $\mathcal{P}$ is Feller, i.e. $\mathcal{P}: C_{b}(G) \rightarrow C_{b}(G)$.

Proof. By continuity of $T_{i}, i \in I$, the update function $\Phi$ is continuous in the first argument. It follows for $f \in C_{b}(G)$ and $x_{n} \rightarrow x$ as $n \rightarrow \infty$ by Lebesgue's Dominated Convergence Theorem

$$
\mathcal{P} f\left(x_{n}\right)=\int_{I} f\left(\Phi\left(x_{n}, u\right)\right) \mathbb{P}^{\xi}(\mathrm{d} u) \rightarrow \int_{I} f(\Phi(x, u)) \mathbb{P}^{\xi}(\mathrm{d} u)=\mathcal{P} f(x) .
$$

Note that $\mathcal{P} f$ is bounded, whenever $f$ is a bounded function.

Theorem 3.5 (almost sure convergence for a compact metric space). Under the standing assumptions, let $T_{i}$ be paracontractive, $i \in I$, and let $(G, d)$ be a compact metric space. Then the sequence $\left(X_{k}\right)$ of random variables generated by Algorithm 1 converges almost surely to a random variable $X_{\mu} \in C$ depending on the initial distribution $\mu$.

Proof. Since $\mathcal{P}$ is Feller and $G$ compact, Theorem A.4 implies that any subsequence of $\left(\nu_{n}\right)$, where $\nu_{n}=\frac{1}{n} \sum_{i=1}^{n} \mathcal{L}\left(X_{i}\right)$, has a convergent subsequence and clusterpoints are invariant measures for $\mathcal{P}$. Let $\left(\nu_{n_{k}}\right)$ be a convergent subsequence with limit $\pi$. So for the bounded and continuous function $\operatorname{dist}(\cdot, C)$ it holds that $\nu_{n_{k}} \operatorname{dist}(\cdot, C) \rightarrow \pi \operatorname{dist}(\cdot, C)=0$ as $k \rightarrow \infty$ by weak convergence of the probability measures and the fact that, by Lemma 3.3, supp $\pi \subset C$.

Due to quasi-nonexpansiveness and Lemma 2.4 (a compact metric space is separable), we have a.s. (for all $\omega \notin N$ with $N$ given by (5)) that $d\left(X_{k+1}, c\right) \leq d\left(X_{k}, c\right)$ for all $c \in C$ and $k \in \mathbb{N}$, which implies $\operatorname{dist}\left(X_{k+1}, C\right) \leq \operatorname{dist}\left(X_{k}, C\right)$ for all $k \in \mathbb{N}$ a.s. It therefore follows that

$$
\mathbb{E}\left[\operatorname{dist}\left(X_{n_{k}}, C\right)\right] \leq \frac{1}{n_{k}} \sum_{i=1}^{n_{k}} \mathbb{E}\left[\operatorname{dist}\left(X_{i}, C\right)\right]=\nu_{n_{k}} \operatorname{dist}(\cdot, C) \rightarrow 0
$$

by monotonicity of $\left(\mathbb{E}\left[\operatorname{dist}\left(X_{k}, C\right)\right]\right)_{k}$. This yields $\mathbb{E}\left[\operatorname{dist}\left(X_{k}, C\right)\right] \rightarrow 0$ as $k \rightarrow \infty$. Now since $\left(\operatorname{dist}\left(X_{k}, C\right)\right)_{k}$ is nonincreasing, it must be that $\operatorname{dist}\left(X_{k}, C\right) \rightarrow 0$ a.s. Hence for any cluster point $x_{\omega}$ of $\left(X_{k}(\omega)\right)_{k}$ we have $x_{\omega} \in C$. This together with a.s. monotonicity of $\left(d\left(X_{k}, c\right)\right)_{k}$ for all $c \in C$ implies that $d\left(X_{k}(\omega), x_{\omega}\right) \rightarrow 0$ for any cluster-point $x_{\omega}$ of $\left(X_{k}(\omega)\right)_{k}$, which implies the uniqueness of $x_{\omega}$. In other words, $\left(X_{k}\right)$ converges almost surely to a random variable $X_{\mu}$, with $X_{\mu}(\omega)=x_{\omega} \in C, \omega \notin N$, as claimed.

\subsection{Finite dimensional normed vector space}

The results for compact metric spaces can be applied, with minor adjustments, to finite dimensional vector spaces. In the following let $(G, d)=(V,\|\cdot\|)$ be a finite dimensional normed vector space over $\mathbb{R}$. This means in particular, that $V$ is also complete and every closed and bounded set is compact (Heine-Borel property) and all norms on $V$ are equivalent. So actually, since all 
$n$-dimensional vector spaces are isomorphic, it is enough to study convergence in $\mathbb{R}^{n}$ equipped with the euclidean norm $\|\cdot\|$.

The following result for $\mathbb{R}^{n}$ is a straight forward application of Theorem 3.5.

Theorem 3.6 (almost sure convergence in $\mathbb{R}^{n}$ ). Under the standing assumptions, let $T_{i}$ : $\mathbb{R}^{n} \rightarrow \mathbb{R}^{n}$ be paracontractive, $i \in I$. Then the sequence $\left(X_{k}\right)$ of random variables generated by Algorithm 1 converges almost surely to a random variable $X_{\mu} \in C$ depending on the initial distribution $\mu$.

Proof. First, suppose $\mu=\delta_{x}$ for $x \in \mathbb{R}^{n}$. Let $N$ be given by (5). The quasi-nonexpansiveness property gives us $\left\|X_{k+1}-c\right\| \leq\left\|X_{k}-c\right\|$ for all $c \in C$ a.s. (i.e. if $\omega \notin N$ ). Letting $c \in C$ with $\operatorname{dist}(x, C)=\|x-c\|$, this implies $X_{k} \in \overline{\mathbb{B}}(c,\|x-c\|)$, where $\overline{\mathbb{B}}(s, \epsilon) \subset \mathbb{R}^{n}$ is the closed ball around $s \in \mathbb{R}^{n}$ with radius $\epsilon$. The assertion $X_{k} \rightarrow X_{\delta_{x}}$ a.s. then follows from Theorem 3.5. Denote the corresponding invariant measure as $\pi_{x}:=\mathcal{L}\left(X_{\delta_{x}}\right)$.

Suppose now that $\mu \in \mathscr{P}\left(\mathbb{R}^{n}\right)$ is arbitrary. For $f \in C_{b}\left(\mathbb{R}^{n}\right)$ one has $p^{k}(x, f) \leq\|f\|_{\infty}$ for all $k \in \mathbb{N}$ and $x \in \mathbb{R}^{n}$. Note that $p^{k}(x, f)=\delta_{x} \mathcal{P}^{k} f$, and from the above argument $\delta_{x} \mathcal{P}^{k} \rightarrow \pi_{x}$ in the weak sense as $k \rightarrow \infty$. Hence by Lebesgue's Dominated Convergence Theorem, we get

$$
\mu \mathcal{P}^{k} f=\int_{\mathbb{R}^{n}} p^{k}(x, f) \mu(\mathrm{d} x) \rightarrow \int_{\mathbb{R}^{n}} \pi_{x} f \mu(\mathrm{d} x)=: \mu \pi_{x} f=: \pi_{\mu} f, \quad \text { as } k \rightarrow \infty .
$$

We conclude that $\mathcal{L}\left(X_{k}\right)=\mu \mathcal{P}^{k} \rightarrow \pi_{\mu}$ weakly. The measure $\pi_{\mu}=\mu \pi_{x}$ is an invariant probability measure for $\mathcal{P}$, since $\pi_{x}$ is a invariant probability measure for $\mathcal{P}$.

Choosing $f=\min \{\operatorname{dist}(\cdot, C), M\} \in C_{b}\left(\mathbb{R}^{n}\right)$ with $M>0$ yields $\mathcal{L}\left(X_{k}\right) f \rightarrow \pi_{\mu} f=0$. Since $f\left(X_{k+1}\right) \leq f\left(X_{k}\right)$ a.s. and $\mathcal{L}\left(X_{k}\right) f=\mathbb{E}\left[f\left(X_{k}\right)\right] \rightarrow 0$, it holds that $f\left(X_{k}\right) \rightarrow 0$ a.s. In particular, $\operatorname{dist}\left(X_{k}, C\right) \rightarrow 0$ a.s. So for a converging subsequence $\left(X_{n_{k}}(\omega)\right)_{k}$ with limit $x_{\omega}$ it holds that $x_{\omega} \in C$. Moreover, since $\left(\left\|X_{k}-x_{\omega}\right\|\right)_{k}$ is monotone, actually $X_{k}(\omega) \rightarrow X_{\mu}(\omega):=\lim _{k} X_{k}(\omega)=$ $x_{\omega} \in C, \omega \notin N$.

\subsection{Weak convergence in Hilbert spaces}

In this section $(G, d)$ is a Hilbert space $(\mathcal{H},\langle\cdot, \cdot\rangle)$. Under the standing assumptions the following extended-valued function

$$
R(x):=\mathbb{E}\left[\left\|x-T_{\xi} x\right\|^{2}\right]=\int_{\Omega}\left\|x-T_{\xi(\omega)} x\right\|^{2} \mathbb{P}(\mathrm{d} \omega)=\int_{I}\left\|x-T_{u} x\right\|^{2} \mathbb{P}^{\xi}(\mathrm{d} u)
$$

is measurable from $\mathcal{H}$ to $[0, \infty]$. Following [5] we use this function to characterize convergence of the consistent fixed point problem under the weaker assumption that the mappings $T_{\xi}$ are averaged (see Eq. (9)).

Lemma 3.7 (properties of $R$ and $C$ for quasi-nonexpansive mappings). In addition to the standing assumptions, suppose that $T_{i}(i \in I)$ is quasi-nonexpansive and continuous. Then

(i) $C=R^{-1}(0)$;

(ii) $R$ is finite everywhere;

(iii) $R$ is continuous;

(iv) $C$ is convex and closed.

Proof. (i) We have $x \in C \Leftrightarrow x \in \operatorname{Fix} T_{\xi}$ a.s. $\Leftrightarrow x=T_{\xi} x$ a.s. $\Leftrightarrow R(x)=0$. 
(ii) Fix $x \in C$, then $x=T_{\xi} x$ a.s. Using quasi-nonexpansivity we get a.s., that

$$
\begin{array}{rlrl}
\left\|y-T_{\xi} y\right\| \leq\|y-x\|+\left\|x-T_{\xi} y\right\| \leq 2\|x-y\| & \forall y \in \mathcal{H}, \\
& \Longleftrightarrow & & \forall y \in \mathcal{H} .
\end{array}
$$

From (11) it follows that $R(y) \leq 4\|y-x\|^{2}<\infty$ for all $y \in \mathcal{H}$.

(iii) Let $x, x_{n} \in \mathcal{H}, n \in \mathbb{N}$, with $x_{n} \rightarrow x$ as $n \rightarrow \infty$. Define the functions $f_{n}(\omega)=$ $\left\|x_{n}-T_{\xi(\omega)} x_{n}\right\|^{2}$ on $\Omega(n \in \mathbb{N})$. Then, by continuity of $T_{\xi(\omega)}$ for fixed $\omega \in \Omega$, one has $f_{n} \rightarrow f:=\left\|x-T_{\xi} x\right\|^{2}$ for all $\omega \in \Omega$. Define the constant function $g(\omega)=8 \epsilon^{2}+8\|x-c\|^{2}$ for some $c \in C$ and some $\epsilon>0$. By (10) we have that $\left\|y-T_{\xi} y\right\| \leq 2\|y-c\|$ for all $y \in \mathcal{H}$. For $y \in \mathbb{B}(x, \epsilon)$ this yields $\left\|y-T_{\xi} y\right\| \leq 2 \epsilon+2\|x-c\|$. We conclude that $g$ is $\mathbb{P}$-integrable and $f_{n} \leq g$ for all $n \in \mathbb{N}$ with $x_{n} \in \mathbb{B}(x, \epsilon)$. Finally, application of Lebesgue's Dominated Convergence Theorem yields $R\left(x_{n}\right)=\mathbb{E} f_{n} \rightarrow \mathbb{E} f=R(x)$ as $n \rightarrow \infty$.

(iv) This follows from [8, Proposition 4.13, Proposition 4.14]. Note that for any $\alpha \in \mathbb{R}, a, b \in \mathcal{H}$ we have [8, Corollary 2.14]

$$
\|\alpha a+(1-\alpha) b\|^{2}=\alpha\|a\|^{2}+(1-\alpha)\|b\|^{2}-\alpha(1-\alpha)\|a-b\|^{2} .
$$

Let $z=\lambda x+(1-\lambda) y$ with $x, y \in R^{-1}(0)=C, \lambda \in[0,1]$. One has with $T_{\xi} x=x$ and $T_{\xi} y=y$ a.s. that a.s. holds

$$
\begin{aligned}
\left\|T_{\xi} z-z\right\|^{2} & =\left\|\lambda\left(T_{\xi} z-x\right)+(1-\lambda)\left(T_{\xi} z-y\right)\right\|^{2} \\
& =\lambda\left\|T_{\xi} z-x\right\|^{2}+(1-\lambda)\left\|T_{\xi} z-y\right\|^{2}-\lambda(1-\lambda)\|x-y\|^{2} \\
& \leq \lambda\|z-x\|^{2}+(1-\lambda)\|z-y\|^{2}-\lambda(1-\lambda)\|x-y\|^{2} \\
& =\|\lambda(z-x)+(1-\lambda)(z-y)\|^{2} \\
& =0 .
\end{aligned}
$$

So $R(z)=0$, i.e. $z \in R^{-1}(0)$. Closedness of $R^{-1}(0)$ follows by continuity of $R$.

In the next theorem we need to compute conditional expectations of nonnegative real-valued random variables, which are non-integrable in general (for example, if the random variable $X_{0}$ with distribution $\mu$ does not have a finite expectation, $\left.\mathbb{E}\left[\left\|X_{0}\right\|\right]=+\infty\right)$. But for these random variables the classical results on integrable random variables are still applicable (see Theorem A.8), also the disintegration theorem is still valid (see Theorem A.9).

The stage is now set to show convergence for the corresponding Markov chain. The next several results concern weak convergence of sequences of random variables with respect to the Hilbert space, namely, $x_{n} \stackrel{\mathrm{w}}{\rightarrow} x$ if $\left\langle x_{n}, y\right\rangle \rightarrow\langle x, y\rangle$ for all $y \in \mathcal{H}$.

Theorem 3.8 (weak cluster points belong to feasible set for averaged mappings). Under the standing assumptions, let $T_{i}$ be $\alpha_{i}$-averaged with $\alpha_{i} \leq \alpha<1$ for all $i \in I$. Then weak cluster points (in the sense of Hilbert spaces) of the sequence $\left(X_{k}\right)_{k \in \mathbb{N}_{0}}$ of random variables in $\mathcal{H}$ generated by Algorithm 1 are a.s. contained in $C$.

Proof. Fix $c \in C$. Since $T_{\xi}$ is averaged we have for all $k \in \mathbb{N}$ that

$$
\left\|X_{k+1}-c\right\|^{2} \leq\left\|X_{k}-c\right\|^{2}-\frac{1-\alpha}{\alpha}\left\|X_{k+1}-X_{k}\right\|^{2}
$$


everywhere but on a $\mathbb{P}$-nullset $N_{c}$, which may depend on $c$. Let $\mathcal{F}_{k}=\sigma\left(X_{0}, \xi_{0}, \ldots, \xi_{k-1}\right)$ be the $\sigma$-algebra of all iterations of the algorithm up to the $k$-th and apply Lemma A.10. We get that $\sum_{k \in \mathbb{N}_{0}} R\left(X_{k}\right)<\infty$ a.s., where from Theorem A.9 follows that $\mathbb{E}\left[\left\|X_{k+1}-X_{k}\right\|^{2} \mid \mathcal{F}_{k}\right]=R\left(X_{k}\right)$. Hence there is $\tilde{N} \subset \Omega$ with $\mathbb{P}(\tilde{N})=0$ and $R\left(X_{k}(\omega)\right) \rightarrow 0$ as $k \rightarrow \infty$ for $\omega \in \Omega \backslash\left(N_{c} \cup \tilde{N}\right)$. By nonexpansiveness of $T_{\xi}$ for all we find for any $x, x_{n} \in \mathcal{H}$

$$
\begin{aligned}
\left\|x-T_{\xi} x\right\|^{2}= & \left\|x_{n}-T_{\xi} x\right\|^{2}+\left\|x-x_{n}\right\|^{2}+2\left\langle x_{n}-T_{\xi} x, x-x_{n}\right\rangle \\
= & \left\|x_{n}-T_{\xi} x\right\|^{2}-\left\|x-x_{n}\right\|^{2}+2\left\langle x-T_{\xi} x, x-x_{n}\right\rangle \\
= & \left\|x_{n}-T_{\xi} x_{n}\right\|^{2}+\left\|T_{\xi} x-T_{\xi} x_{n}\right\|^{2}+2\left\langle x_{n}-T_{\xi} x_{n}, T_{\xi} x_{n}-T_{\xi} x\right\rangle \\
& \quad-\left\|x-x_{n}\right\|^{2}+2\left\langle x-T_{\xi} x, x-x_{n}\right\rangle \\
\leq & \left\|x_{n}-T_{\xi} x_{n}\right\|^{2}+2\left\langle x_{n}-T_{\xi} x_{n}, T_{\xi} x_{n}-T_{\xi} x\right\rangle+2\left\langle x-T_{\xi} x, x-x_{n}\right\rangle \\
\leq & \left\|x_{n}-T_{\xi} x_{n}\right\|^{2}+2\left\|x_{n}-T_{\xi} x_{n}\right\|\left\|x_{n}-x\right\|+2\left\langle x-T_{\xi} x, x-x_{n}\right\rangle .
\end{aligned}
$$

Taking expectation and using Jensen's inequality yields

$$
R(x) \leq R\left(x_{n}\right)+2 \sqrt{R\left(x_{n}\right)}\left\|x_{n}-x\right\|+2 \mathbb{E}\left[\left\langle x-T_{\xi} x, x-x_{n}\right\rangle\right] .
$$

Now assume that the sequence $\left(x_{n}\right)$ is weakly converging to $x \in \mathcal{H}$, i.e. $x_{n} \stackrel{\mathrm{w}}{\rightarrow} x$. Then the functions $f_{n}=\left\langle x-T_{\xi} x, x-x_{n}\right\rangle, n \in \mathbb{N}$, on $\Omega$ satisfy $f_{n} \rightarrow 0$ a.s. Defining the $\mathbb{P}$-integrable function $g(\omega):=\left\|x-T_{\xi(\omega)} x\right\| \sup _{n}\left\|x-x_{n}\right\|$ gives us $\left|f_{n}\right| \leq g$ for all $n \in \mathbb{N}$ and hence by Lebesgue's Dominated Convergence Theorem $\mathbb{E}\left[\left\langle x-T_{\xi} x, x-x_{n}\right\rangle\right] \rightarrow 0$ as $n \rightarrow \infty$.

So for $\omega \in \Omega \backslash\left(N_{c} \cup \tilde{N}\right)$ there is a weakly convergent subsequence of the bounded sequence $\left(X_{k}(\omega)\right)_{k \in \mathbb{N}}$, denoted $x_{n}:=X_{k_{n}}(\omega) \stackrel{\mathrm{w}}{\rightarrow} x_{\omega}=: x$ as $n \rightarrow \infty$. As shown above this subsequence satisfies $R\left(x_{n}\right) \rightarrow 0$ as $n \rightarrow \infty$. We conclude with (13) that $R(x)=0$, i.e. $x \in C$ and hence any weak cluster point of the sequence $\left(X_{k}(\omega)\right)_{k}$ is contained in $C$.

In the case of separable Hilbert spaces, we are able to show Fejér monotonicity of the sequence $\left(X_{k}\right)$ a.s., so the classical theory of convergence analysis from [8] can be applied in this case. An analogous statement for nonseparable Hilbert spaces remains open since we do not have the representation Lemma 2.4 at hand.

Theorem 3.9 (almost sure weak convergence under separability). Under the same assumptions as in Theorem 3.8 assume additionally that $\mathcal{H}$ is a separable Hilbert space. Then the sequence $\left(X_{k}\right)$ is a.s. weakly convergent (in the sense of Hilbert spaces) to a random variable $X_{\mu} \in C$, depending on the initial distribution $\mu$. Furthermore $P_{C} X_{k} \rightarrow X_{\mu}$ strongly a.s. as $k \rightarrow \infty$.

Proof. Instead of a nullset $N_{c}$, which may depend on $c \in C$, as in the proof of Theorem 3.8, separability gives with help of Lemma 2.4 that there is a nullset $N$, such that on $\Omega \backslash N$ Eq. (12) is satisfied for all $c \in C$. This implies a.s. Fejér monotonicity of $\left(X_{k}\right)$. Since from Theorem 3.8 follows that weak clusterpoints of $\left(X_{k}\right)$ are contained in $C$ a.s., we can now apply Theory in [8] developed for Fejér monotone sequences, we get: From [8, Theorem 5.5] (a Fejér monotone sequence w.r.t. $C$ that has all weak clusterpoints in $C$ is weakly convergent to a point in $C$ ) follows that $X_{k} \stackrel{\mathrm{w}}{\rightarrow} X_{\mu} \in C$ a.s.

For strong convergence of $\left(P_{C} X_{k}\right)$ a.s. we apply [8, Proposition 5.7]. From [8, Corollary 5.8] we get from $X_{k} \stackrel{\mathrm{w}}{\rightarrow} X_{\mu}$ a.s., that $P_{C} X_{k} \rightarrow X_{\mu}$ a.s. strongly as $k \rightarrow \infty$.

Example 3.10 (convergence to projection for affine subspaces). Let $\mathcal{H}$ be separable and $C_{i}$ be an affine subspace, $i \in I$, where $I$ is an arbitrary index set. Let $T_{i}=P_{i}$ be the projector onto 
$C_{i}$. Under the standing assumptions holds that $\lim _{k} X_{k}=X_{\mu}=P_{C} X_{0}$ for $X_{0} \sim \mu$ and any $\mu \in \mathscr{P}(\mathcal{H})$.

We show, that $P_{C} X_{k+1}=P_{C} X_{k}$ for any $k \in \mathbb{N}_{0}$. This allows us to conclude that $P_{C} X_{k}=$ $P_{C} X_{0}$ for any $k \in \mathbb{N}_{0}$, and thus $P_{C} X_{0}$ is the only possible weak cluster point of $\left(X_{k}\right)$ by Theorem 3.9. Using the characterization [15, Theorem 4.1] (if $K \subset \mathcal{H}$ is nonempty, closed and convex and $u \in K$ then $\langle x-u, k-u\rangle \leq 0$ for all $k \in K$ iff $\left.u=P_{K} x\right)$ of a projection, we find with help of [15, Theorem 4.9] (for a subspace $S$ holds that $\left\langle x-P_{S} x, s\right\rangle=0$ for all $s \in S$ ), that for $c \in C$ holds that

$$
\left\langle X_{k+1}-P_{C} X_{k}, c-P_{C} X_{k}\right\rangle=\underbrace{\left\langle P_{\xi_{k}} X_{k}-X_{k}, c-P_{C} X_{k}\right\rangle}_{=0}+\underbrace{\left\langle X_{k}-P_{C} X_{k}, c-P_{C} X_{k}\right\rangle}_{\leq 0} \leq 0 .
$$

Hence by $\left[15\right.$, Theorem 4.1] we have that $P_{C} X_{k+1}=P_{C} X_{k}$.

\subsection{Linear rates of convergence}

We will assume in this section that $\mathcal{H}$ is a separable Hilbert space and $T_{i}$ is $\alpha_{i}$-averaged, $i \in I$. We will furthermore assume, that $\alpha_{i} \leq \alpha$ for some $\alpha<1$. As with the deterministic case, geometric convergence of the algorithm can be analyzed by introducing a condition on the set of fixed points. In the context of set feasibility with finitely many sets, the condition is equivalent to linear regularity of the sets [2, Assumption 2]: There exists $\kappa>0$ such that

$$
\operatorname{dist}^{2}(x, C) \leq \kappa R(x) \quad \forall x \in \mathcal{H} .
$$

In the more general context of fixed point mappings, this property is more appropriately called global metric subregularity of $R$ at all points in $C$ for 0 [16]; in particular there exists a $\kappa>0$ such that

$$
\operatorname{dist}^{2}\left(x, R^{-1}(0)\right) \leq \kappa R(x) \quad \forall x \in \mathcal{H} .
$$

Here $C=R^{-1}(0)$, so the above is just another way of writing (14). The smallest constant satisfying this inequality will be called the regularity constant, it is given by

$$
\sup _{x \in \mathcal{H} \backslash C} \frac{\operatorname{dist}^{2}(x, C)}{R(x)} .
$$

Theorem 3.11. In addition to the standing assumptions, suppose the regularity condition in Eq. (14) is satisfied and $T_{i}$ is $\alpha_{i}$-averaged, $i \in I$ with $\alpha_{i} \leq \alpha$ for some $\alpha<1$. Then the RFI converges geometrically in expectation to the fixed point set, i.e. for any initial distribution

$$
\mathbb{E}\left[\operatorname{dist}\left(X_{k+1}, C\right)\right] \leq \sqrt{1-\kappa^{-1} \frac{1-\alpha}{\alpha}} \mathbb{E}\left[\operatorname{dist}\left(X_{k}, C\right)\right] \quad \forall k \in \mathbb{N}_{0} .
$$

Proof. Revisiting (12) in the proof of Theorem 3.8 gives us for $\omega \in \Omega \backslash N$ ( $N$ given by (5)) and $x=P_{C} X_{k}(\omega)$

$$
\operatorname{dist}^{2}\left(X_{k+1}(\omega), C\right) \leq\left\|X_{k+1}(\omega)-x\right\|^{2} \leq \operatorname{dist}^{2}\left(X_{k}(\omega), C\right)-\frac{1-\alpha}{\alpha}\left\|X_{k+1}(\omega)-X_{k}(\omega)\right\|^{2} .
$$

With help of Jensen's inequality and concavity of $x \mapsto \sqrt{x}$ on $[0, \infty)$, we get that

$$
\begin{aligned}
\mathbb{E}\left[\operatorname{dist}\left(X_{k+1}, C\right) \mid \mathcal{F}_{k}\right] & \leq \mathbb{E}\left[\sqrt{\operatorname{dist}^{2}\left(X_{k}, C\right)-\frac{1-\alpha}{\alpha}\left\|T_{\xi_{k}} X_{k}-X_{k}\right\|^{2}} \mid \mathcal{F}_{k}\right] \\
& \leq \sqrt{\operatorname{dist}^{2}\left(X_{k}, C\right)-\frac{1-\alpha}{\alpha} \mathbb{E}\left[\left\|T_{\xi_{k}} X_{k}-X_{k}\right\|^{2} \mid \mathcal{F}_{k}\right]}
\end{aligned}
$$




$$
\begin{aligned}
& =\sqrt{\operatorname{dist}^{2}\left(X_{k}, C\right)-\frac{1-\alpha}{\alpha} R\left(X_{k}\right)} \\
& \leq \sqrt{1-\kappa^{-1} \frac{1-\alpha}{\alpha}} \operatorname{dist}\left(X_{k}, C\right) .
\end{aligned}
$$

Note that it could be $\mathbb{E}\left[\operatorname{dist}\left(X_{k}, C\right)\right]=\infty$ for all $k \in \mathbb{N}$, depending on the initial distribution $\mu$.

The next theorem concerns the Wasserstein distance of two probability measures. For two measures $\nu_{1}, \nu_{2} \in \mathscr{P}(G)$ this is given by

$$
W\left(\nu_{1}, \nu_{2}\right)=\inf _{\substack{Y_{1} \sim \nu_{1} \\ Y_{2} \sim \nu_{2}}} \mathbb{E}\left[\left\|Y_{1}-Y_{2}\right\|\right]
$$

Theorem 3.12 (strong convergence and geometric convergence of measures). Under the standing assumptions, suppose the regularity condition in Eq. (14) is satisfied and $T_{i}$ is $\alpha_{i}$-averaged, $i \in I$ with $\alpha_{i} \leq \alpha$ for some $\alpha<1$. Then $X_{k} \rightarrow X$ strongly a.s. as $k \rightarrow \infty$ and the Wasserstein distances $W\left(\mathcal{L}\left(X_{k}\right), \mathcal{L}(X)\right)$ also converge geometricly, there is $r \in(0,1)$ such that

$$
W\left(\mathcal{L}\left(X_{k}\right), \mathcal{L}(X)\right) \leq 2 r^{k} W\left(\mathcal{L}\left(X_{0}\right), \mathcal{L}(X)\right) .
$$

Proof. See also [8, Theorem 5.12]. One has a.s. that

$$
\left\|X_{k}-X_{k+m}\right\| \leq\left\|X_{k}-P_{C} X_{k}\right\|+\left\|P_{C} X_{k}-X_{k+m}\right\| \leq 2 \operatorname{dist}\left(X_{k}, C\right) \leq 2 \sqrt{\kappa R\left(X_{k}\right)} .
$$

We used here, that $T_{\xi}$ is nonexpansive and it satisfies $T_{\xi} c=c$ for any $c \in C$ a.s., hence $\left\|P_{C} X_{k}-X_{k+m}\right\|=\left\|T_{\xi_{k+m-1}} \cdots T_{\xi_{k}} P_{C} X_{k}-X_{k+m}\right\| \leq \operatorname{dist}\left(X_{k}, C\right)$. This gives us that $\left(X_{k}\right)$ is a Cauchy sequence a.s., since $R\left(X_{k}\right) \rightarrow 0$ as seen in the proof of Theorem 3.8. It's limit $X$ is contained in $C$, since it's weak limit needs to coincide with the strong limit. Letting $m \rightarrow \infty$ one arrives at $\left\|X_{k}-X\right\| \leq 2 \operatorname{dist}\left(X_{k}, C\right)$. Taking the expectation yields $\mathbb{E}\left[\left\|X_{k}-X\right\|\right] \leq$ $2 \mathbb{E}\left[\operatorname{dist}\left(X_{k}, C\right)\right]$. Hence, using Theorem 3.11 gives us $\mathbb{E}\left[\left\|X_{k}-X\right\|\right] \leq 2 r^{k} \mathbb{E}\left[\operatorname{dist}\left(X_{0}, C\right)\right]$ with $r=\sqrt{1-\kappa^{-1} \frac{1-\alpha}{\alpha}}$ and using the fact that $\mathbb{E}\left[\operatorname{dist}\left(X_{0}, C\right)\right] \leq W\left(\mathcal{L}\left(X_{0}\right), \mathcal{L}(X)\right)$, we have, by the definition of the Wasserstein distance,

$$
W\left(\mathcal{L}\left(X_{k}\right), \mathcal{L}(X)\right) \leq 2 r^{k} W\left(\mathcal{L}\left(X_{0}\right), \mathcal{L}(X)\right) .
$$

Note that it could be $W\left(\mathcal{L}\left(X_{0}\right), \mathcal{L}(X)\right)=\infty$, depending on the initial distribution $\mu$.

Remark 3.13 ( $\epsilon$-fixed point): In order to assure that, with probability greater than $1-\beta$, the $k$ th iterate is in an $\epsilon$ neighborhood of the feasible set $C$, it is sufficient that $k \geq \ln \left(\frac{\beta \epsilon}{\sqrt{\kappa R(x)}}\right) / \ln (c)$, where $c=\sqrt{1-\frac{1-\alpha}{\alpha} \kappa^{-1}}$ and $X_{0} \sim \delta_{x}$. To see this, note that, by Markov's inequality,

$$
\begin{aligned}
\mathbb{P}\left(X_{k} \in C+\epsilon \mathbb{B}(0,1)\right) & =\mathbb{P}\left(\operatorname{dist}\left(X_{k}, C\right)<\epsilon\right) \\
& =1-\mathbb{P}\left(\operatorname{dist}\left(X_{k}, C\right) \geq \epsilon\right) \\
& \geq 1-\frac{\mathbb{E}\left[\operatorname{dist}\left(X_{k}, C\right)\right]}{\epsilon} \\
& \geq 1-r^{k} \frac{\operatorname{dist}(x, C)}{\epsilon} \\
& \geq 1-r^{k} \frac{\sqrt{\kappa R(x)}}{\epsilon} .
\end{aligned}
$$


Remark 3.14: As seen in Example 3.10 the probability $\mathbb{P}\left(X_{k} \in C\right)$ can increase to 1 as $k \rightarrow \infty$, but this is not necessarily the case, as we will see in Examples 4.7 and 4.9. There, one finds that $\mathbb{P}\left(X_{k} \in C\right)=\mathbb{P}\left(X_{0} \in C\right)$ for $k \in \mathbb{N}$. In Example 4.8 it holds that $\mathbb{P}\left(X_{k} \in C\right)=\mathbb{P}\left(X_{1} \in C\right)$ for all $k \in \mathbb{N}$.

Theorem 3.15 (necessary and sufficient conditions for geometric convergence). Under the standing assumptions, let $T_{i}$ be $\alpha_{i}$-averaged, $i \in I$ with $\alpha_{i} \leq \alpha$ for some $\alpha<1$. The regularity condition in Eq. (14) is satisfied if and only if there exists $r \in[0,1)$ such that

$$
\mathbb{E}\left[\operatorname{dist}\left(T_{\xi} x, C\right)\right] \leq r \operatorname{dist}(x, C) \quad \forall x \in \mathcal{H} .
$$

Furthermore, condition Eq. (14) is necessary and sufficient for geometric convergence in expectation of Algorithm 1 to the fixed point set $C$ as in Eq. (15) with a uniform constant for all initial probability measures.

Proof. Eq. (14) implies Eq. (15), which in turn implies Eq. (16) (with $X_{0} \sim \delta_{x}$ ) by Theorem 3.11 with $r=\sqrt{1-\kappa^{-1} \frac{1-\alpha}{\alpha}}$. The other implication follows the same proof pattern as [6, Theorem 3.11]. We note that, by Theorem A.9, if $X_{0} \sim \delta_{x}$ for $x \in \mathcal{H}$, then

$$
\mathbb{E}\left[\left\|X_{1}-X_{0}\right\| \mid \xi_{0}\right]=\left\|T_{\xi_{0}} x-x\right\|,
$$

hence by Hölder's inequality

$$
\mathbb{E}\left[\left\|X_{1}-X_{0}\right\|\right] \leq \sqrt{R(x)}
$$

Furthermore we can estimate

$$
\left\|X_{1}-X_{0}\right\|=\left\|X_{1}-P_{C} X_{1}+P_{C} X_{1}-X_{0}\right\| \geq \operatorname{dist}\left(X_{0}, C\right)-\operatorname{dist}\left(X_{1}, C\right) .
$$

Taking the expectation above, the assumption that $\mathbb{E}\left[\operatorname{dist}\left(X_{1}, C\right)\right] \leq r \mathbb{E}\left[\operatorname{dist}\left(X_{0}, C\right)\right]$ yields

$$
(\forall x \in \mathcal{H}) \quad R(x) \geq(1-r)^{2} \operatorname{dist}^{2}(x, C),
$$

i.e. the constant $\kappa$ in Eq. (14) is finite with $\kappa \leq(1-r)^{-2}<\infty$. So Eq. (16) implies Eq. (14).

For the last implication of the theorem, note that, in case Eq. (15) is satisfied with the same constant $r \in(0,1)$ for all Dirac measures $\delta_{x}$ with $x \in \mathcal{H}$, then Eq. (16) also holds (letting $X_{0} \sim \delta_{x}$ ) and hence by the above equivalence Eq. (14) is satisfied. This completes the proof.

Remark 3.16: Conventional analytical strategies invoke strong convexity in order to achieve geometric convergence. Our analysis makes no such assumption on the sets $C_{i}$. Theorem 3.15 shows that geometric convergence is a by-product, mainly, of the regularity of the set of fixed points. The results of [6] indicate that one could formulate a necessary regularity condition for sublinear convergence, which also might be useful for stochastic algorithms.

\section{Applications}

We specialize the framework above to several well-known settings: consistent convex feasibility, linear operator equations and in particular Hilbert-Schmidt operators (i.e. linear integral equations). 


\subsection{Feasibility and stochastic projections}

There are many algorithms for solving convex feasibility problems. We focus on the (conceptually) simplest of these, namely stochastic projections. In the context of Algorithm $1, T_{i}=P_{i}$ is a projector, $i \in I$, onto a nonempty closed and convex set $C_{i} \subset \mathcal{H}, i \in I$ and $\mathcal{H}$ a Hilbert space. Note that projectors are $\frac{1}{2}$-averaged operators [8, Proposition 4.8] (also referred to as firmly nonexpansive operators), so $\alpha_{i}=\frac{1}{2}$ for all $i \in I$, we then can choose the upper bound $\alpha=\frac{1}{2}$ as well. Also note that Fix $P_{i}=C_{i}, i \in I$.

As a first assertion we give an equivalent characterization for the regularity property in Eq. (14) using just properties of $R$. This characterization, known as Kurdyka-Łojasiewicz (KL) property, eliminates the term with the distance to the usually unknown fixed point set $C$, but one needs to be able to compute the first derivative of the function $R$. For convex sets this is unproblematic since $R$ is the expectation of the squared distances to the convex sets $C_{i}$, see Lemma A.11.

Definition 4.1 (KL property). A convex, continuously differentiable function $f: \mathcal{H} \rightarrow \mathbb{R}$ with $\inf _{x} f(x)=0$ and $S:=\operatorname{argmin} f \neq \emptyset$ is said to have the global KL property, if there exists a concave continuously differentiable function $\varphi: \mathbb{R}_{+} \rightarrow \mathbb{R}_{+}$with $\varphi(0)=0$ and $\varphi^{\prime}>0$ such that

$$
\varphi^{\prime}(f(x))\|\nabla f(x)\| \geq 1 \quad \forall x \in \mathcal{H} \backslash S .
$$

The following theorem is a direct consequence of [17].

Proposition 4.2 (equivalent characterization of Eq. (14)). Under the standing assumptions, let $T_{i}=P_{i}$ be projectors onto nonempty, closed and convex sets, $i \in I$. Then the regularity condition in Eq. (14) is satisfied with $\kappa>0$ if and only if $R(x) \leq \frac{\kappa}{4}\|\nabla R(x)\|^{2} \forall x \in \mathcal{H}$, i.e. $R$ has the global KL property.

Proof. Apply [17, Corollary 6] with $\varphi(s):=\sqrt{\kappa s}$ and $f=R$ and note that $R$ is convex and differentiable (see Lemma A.11).

Theorem 4.3 (uniform bounds). Under the standing assumptions, suppose the regularity condition in Eq. (14) is satisfied and that $\mathcal{H}$ is separable and $T_{i}=P_{i}$ are projectors onto nonempty, closed and convex sets, $i \in I$. Then the probability of any point being feasible is uniformly bounded, i.e. $\mathbb{P}\left(x \in C_{\xi}\right) \leq r<1$ for all $x \in \mathcal{H} \backslash C$.

Proof. It holds surely for all $x \in \mathcal{H}$

$$
\operatorname{dist}\left(P_{\xi} x, C\right) \geq \operatorname{dist}(x, C)-\operatorname{dist}\left(x, C_{\xi}\right) .
$$

This, together with the expectation

$$
\mathbb{E}\left[\operatorname{dist}\left(x, C_{\xi}\right)\right]=\mathbb{E}\left[\operatorname{dist}\left(x, C_{\xi}\right) \mathbb{1}_{\left\{x \notin C_{\xi}\right\}}\right] \leq \mathbb{E}\left[\operatorname{dist}(x, C) \mathbb{1}_{\left\{x \notin C_{\xi}\right\}}\right]=\operatorname{dist}(x, C)\left(1-\mathbb{P}\left(x \in C_{\xi}\right)\right)
$$

yields, for $X_{0} \sim \delta_{x}$,

$$
\mathbb{E}\left[\operatorname{dist}\left(X_{1}, C\right)\right] \geq \mathbb{P}\left(x \in C_{\xi}\right) \operatorname{dist}(x, C) .
$$

Hence by Theorem 3.15

$$
1>r:=\sup _{x \in \mathcal{H} \backslash C} \frac{\mathbb{E}\left[\operatorname{dist}\left(X_{1}, C\right)\right]}{\operatorname{dist}(x, C)} \geq \sup _{x \in \mathcal{H} \backslash C} \mathbb{P}\left(x \in C_{\xi}\right) .
$$


Theorem 4.4 (finite vs. infinite convergence). Under the standing assumptions, let $\mathcal{H}$ be separable and let $T_{i}=P_{i}$ be projectors $(i \in I)$. Then one of the following holds:

(i) $\mathbb{P}\left(X_{1} \in C\right)=1$ and $\mathbb{P}\left(X_{n} \in C\right)=1$ for all $n \in \mathbb{N}$,

(ii) $\mathbb{P}\left(X_{1} \in C\right)<1$ and $\mathbb{P}\left(X_{n} \in C\right)<1$ for all $n \in \mathbb{N}$.

Proof. (i) If $\mathbb{P}\left(X_{1} \in C\right)=1$, then $X_{k}=X_{1}$ a.s. for all $k \geq 1$.

(ii) From $\int p(x, C) \mu(\mathrm{d} x)=\mathbb{P}\left(X_{1} \in C\right)<1$ we get, that there is $x \in \operatorname{supp} \mu \backslash C$ with $p(x, C)<$ 1 , where $\mu$ is the initial distribution. Since $p(x, \mathcal{H} \backslash C)>0$, there exists $y \in \operatorname{supp} p(x, \cdot) \backslash C$. Then by Theorem A.3 this implies that $p(x, \overline{\mathbb{B}}(y, \epsilon))>0$ for all $\epsilon>0$.

Furthermore, one has for any $\epsilon>0$ that

$$
(\forall z \in \overline{\mathbb{B}}(y, \epsilon)) \quad p(z, \overline{\mathbb{B}}(y, 2 \epsilon)) \geq p(x, \overline{\mathbb{B}}(y, \epsilon))>0 .
$$

To see this, note that, for $\omega \in M(\epsilon):=\left\{\omega \in \Omega \mid P_{\xi(\omega)} x \in \overline{\mathbb{B}}(y, \epsilon)\right\}$, we have

$$
\begin{aligned}
\left\|P_{\xi(\omega)} z-y\right\| & \leq\left\|P_{\xi(\omega)} z-P_{\xi(\omega)} y\right\|+\left\|P_{\xi(\omega)} y-y\right\| \\
& \leq\|z-y\|+\left\|P_{\xi(\omega)} y-y\right\| \\
& \leq\|z-y\|+\left\|P_{\xi(\omega)} x-y\right\| \\
& \leq 2 \epsilon .
\end{aligned}
$$

Here we have used nonexpansiveness of $P_{\xi}$ and the definition of a projection. Now (17) follows from the identity $\mathbb{P}\left(M_{k}(\epsilon)\right)=p(x, \overline{\mathbb{B}}(y, \epsilon))>0$.

Furthermore, one has for $\epsilon>0$ that

$$
(\forall w \in \overline{\mathbb{B}}(x, \epsilon)) \quad p(w, \overline{\mathbb{B}}(y, 2 \epsilon)) \geq p(x, \overline{\mathbb{B}}(y, \epsilon))>0 .
$$

To see this, note that for $\omega \in M(\epsilon)$, we have

$$
\begin{aligned}
\left\|P_{\xi(\omega)} w-y\right\| & \leq\left\|P_{\xi(\omega)} w-P_{\xi(\omega)} x\right\|+\left\|P_{\xi(\omega)} x-y\right\| \\
& \leq 2 \epsilon .
\end{aligned}
$$

Now, fix $\epsilon>0$ such that both $\overline{\mathbb{B}}(y, \epsilon) \cap C=\emptyset$ and $\overline{\mathbb{B}}(x, \epsilon) \cap C=\emptyset$. We get for any $w \in \mathcal{H}$ and $n \in \mathbb{N}$

$$
p^{n+1}(w, \overline{\mathbb{B}}(y, \epsilon)) \geq \int_{\overline{\mathbb{B}}\left(y, \frac{\epsilon}{2}\right)} p(z, \overline{\mathbb{B}}(y, \epsilon)) p^{n}(w, \mathrm{~d} z) \geq p\left(x, \overline{\mathbb{B}}\left(y, \frac{\epsilon}{2}\right)\right) p^{n}\left(w, \overline{\mathbb{B}}\left(y, \frac{\epsilon}{2}\right)\right) .
$$

So iteratively, denoting $\epsilon_{n}:=2^{-n} \epsilon$, we arrive at

$$
p^{n+1}(w, \overline{\mathbb{B}}(y, \epsilon)) \geq \prod_{i=1}^{n} p\left(x, \overline{\mathbb{B}}\left(y, \epsilon_{i}\right)\right) p\left(w, \overline{\mathbb{B}}\left(y, \epsilon_{n}\right)\right) .
$$

The last probability can be estimated for $w \in \overline{\mathbb{B}}\left(x, \epsilon_{n+1}\right)$ by (18) through

$$
p\left(w, \overline{\mathbb{B}}\left(y, \epsilon_{n}\right)\right) \geq p\left(x, \overline{\mathbb{B}}\left(y, \epsilon_{n+1}\right)\right) .
$$


Summarizing, we have that $p^{n}(w, \overline{\mathbb{B}}(y, \epsilon))$ is locally uniformly bounded from below for $w \in \overline{\mathbb{B}}\left(x, \epsilon_{n}\right)$. That implies

$$
\begin{aligned}
\mathbb{P}\left(X_{n} \in \mathcal{H} \backslash C\right) & =\int_{\mathcal{H}} p^{n}(w, \mathcal{H} \backslash C) \mu(\mathrm{d} w) \\
& \geq \int_{\overline{\mathbb{B}}\left(x, \epsilon_{n}\right)} p^{n}(w, \overline{\mathbb{B}}(y, \epsilon)) \mu(\mathrm{d} w) \\
& \geq\left[p\left(x, \overline{\mathbb{B}}\left(y, \epsilon_{n}\right)\right)\right]^{n} \mu\left(\overline{\mathbb{B}}\left(x, \epsilon_{n}\right)\right)>0,
\end{aligned}
$$

i.e. $\mathbb{P}\left(X_{n} \in C\right)<1$ for all $n \in \mathbb{N}$, as claimed.

Remark 4.5: Theorem 4.4 can be interpreted as a lower bound on the complexity of the RFI analogous to the deterministic case [6, Theorem 5.2], where the alternating projection algorithm converges either after one iteration or after infinitely many. Alternatively, the stopping or hitting time of a process is defined as

$$
T:=\inf \left\{n \mid X_{n} \in C\right\} .
$$

In this context, Theorem 4.4 says that, either $\mathbb{P}(T=1)=1$ or $\mathbb{P}(T=n)<1$ for all $n \in \mathbb{N}$. Note, it could happen that $\mathbb{P}(T=\infty)=1$, in which case $\mathbb{P}(T=n)=0$ for all $n \in \mathbb{N}$.

Example 4.6 (finite and infinite convergence). With just two sets, the deterministic alternating projections algorithms can converge in finitely many steps. But when the projections onto the respective sets are randomly selected, convergence might only come after infinitely many steps. For example, let $C_{1}=\mathbb{R}_{+} \times \mathbb{R}$ and $C_{2}=\mathbb{R} \times \mathbb{R}_{+}$and $\mathbb{P}(\xi=1)=0.3, \mathbb{P}(\xi=2)=0.7$. Then $C=\mathbb{R}_{+} \times \mathbb{R}_{+}$. Set $\mu=\delta_{x}$, where $x=\left(\begin{array}{l}-1 \\ -1\end{array}\right)$. Then $\mathbb{P}\left(X_{1} \in C\right)=0$ or more generally $\mathbb{P}\left(X_{n} \in C\right)=1-0.3^{n}-0.7^{n}<1, n \in \mathbb{N}$. Now let $\mathbb{P}(\xi=1)=1$ and $\mathbb{P}(\xi=2)=0$, then $C=C_{1}$ and for $\mu$ as above $\mathbb{P}\left(X_{1} \in C\right)=1$ and so $\mathbb{P}\left(X_{n} \in C\right)=1$.

Example 4.7 (no uniform geometric convergence). In this example we show a sublinear convergence rate for infinitely many overlapping intervals. This is in contrast to the convergence properties of finitely many intervals with nonempty interior, where one would expect a geometric rate.

Let $\xi \sim \operatorname{unif}\left[\epsilon-\frac{1}{2}, \frac{1}{2}-\epsilon\right]$ for some $\epsilon \in\left[0, \frac{1}{2}\right)$. Define the nonempty and closed intervals $C_{r}=\left[r-\frac{1}{2}, r+\frac{1}{2}\right], r \in \mathbb{R}$.

$$
\begin{aligned}
C_{r} & =\left[r-\frac{1}{2}, r+\frac{1}{2}\right] \\
r & \in[-0.3,0.3] \\
C & =[-0.2,0.2]
\end{aligned}
$$

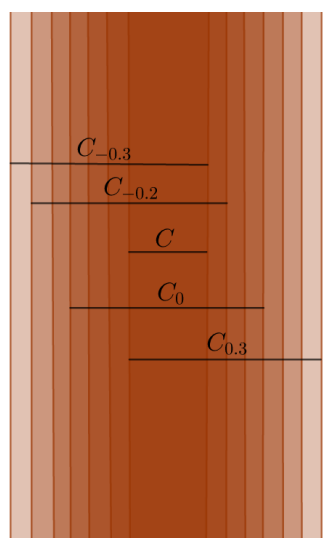

Figure 1

The projector onto these intervals is given by

$$
P_{r} x=\left\{\begin{array}{ll}
r+\frac{1}{2} & x \geq r+\frac{1}{2} \\
r-\frac{1}{2} & x \leq r-\frac{1}{2} \\
x & r-\frac{1}{2} \leq x \leq r+\frac{1}{2}
\end{array} .\right.
$$


The Lebesgue-density $\rho_{\epsilon}$ of $\xi$ is

$$
\rho_{\epsilon}(y)=\frac{1}{1-2 \epsilon} \mathbb{1}_{\left[\epsilon-\frac{1}{2}, \frac{1}{2}-\epsilon\right]}(y) .
$$

One can compute

$$
\begin{aligned}
R_{\epsilon}(x) & =\mathbb{E}\left[\left|P_{\xi} x-x\right|^{2}\right]=\int_{\mathbb{R}}\left|P_{r} x-x\right|^{2} \rho_{\epsilon}(r) \mathrm{d} r=\frac{1}{1-2 \epsilon} \int_{\epsilon-\frac{1}{2}}^{\frac{1}{2}-\epsilon}\left|P_{r} x-x\right|^{2} \mathrm{~d} r \\
& =\frac{1}{1-2 \epsilon} \mathbb{1}_{[\epsilon, \infty)}(|x|) \frac{(|x|-\epsilon)^{3}+\min (1-|x|-\epsilon, 0)^{3}}{3} .
\end{aligned}
$$

Now, let us examine regularity properties. For the case $\epsilon \in\left[0, \frac{1}{2}\right)$, the problem is a consistent feasibility problem with $C=[-\epsilon, \epsilon]$. While the regularity condition in Eq. (14) is trivially satisfied for $|x| \leq \epsilon$, for $\epsilon \leq|x| \leq 1-\epsilon$ we find $R_{\epsilon}(x)=\frac{1}{1-2 \epsilon} \frac{(|x|-\epsilon)^{3}}{3}$ and $\operatorname{dist}^{2}(x, C)=(|x|-\epsilon)^{2}$. So the regularity property in Eq. (14) is not satisfied for any $\kappa>0$ here. That means by Theorem 3.15, that we cannot expect uniform geometric convergence (i.e. there is no $r \in[0,1$ ) with $\mathbb{E}\left[\operatorname{dist}\left(X_{k+1}, C\right)\right] \leq r \mathbb{E}\left[\operatorname{dist}\left(X_{k}, C\right)\right]$, where $\left.X_{0} \sim \delta_{x}, x \in \mathcal{H}\right)$.

Example 4.8 (uniform geometric convergence). We provide here a concrete example where geometric convergence of the RFI is achieved. This is somewhat surprising since the angle between the sets can become arbitrarily small. In the deterministic setting, this results in arbitrarily slow convergence of the algorithm. This provides some intuition for why stochastic algorithms can outperform deterministic variants.

Let $C_{\alpha}:=\mathbb{R} e_{\alpha}$ with $e_{\alpha}=\left(\begin{array}{c}\cos (\alpha) \\ \sin (\alpha)\end{array}\right), \alpha \in[0,2 \pi)$ and let $\xi \sim$ unif $[0, \beta]$, where $\beta \in\left(0, \frac{\pi}{2}\right)$.

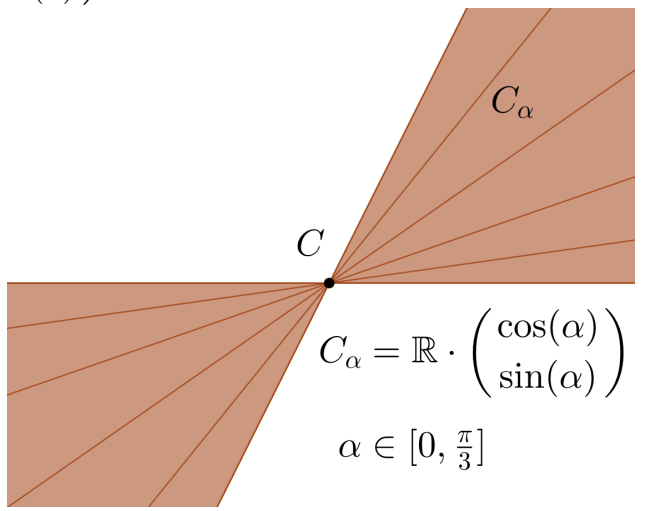

Figure 2

We have $C=\{0\}$, so $\operatorname{dist}(x, C)=\|x\|$ and the density of $\xi$ is $\rho_{\beta}(\alpha)=\frac{1}{\beta} \mathbb{1}_{[0, \beta]}(\alpha)$. The projector onto the linear subspace $C_{\alpha}$ of $\mathbb{R}^{2}$ is given by

$$
P_{\alpha}(x)=x-\left\langle\left(\begin{array}{c}
\sin (\alpha) \\
-\cos (\alpha)
\end{array}\right), x\right\rangle\left(\begin{array}{c}
\sin (\alpha) \\
-\cos (\alpha)
\end{array}\right) .
$$

We find then

$$
\begin{aligned}
R_{\beta}(x) & =\frac{1}{\beta} \int_{0}^{\beta}\left\|P_{\alpha} x-x\right\|^{2} \mathrm{~d} \alpha=\frac{1}{\beta} \int_{0}^{\beta}\left(x_{1} \sin (\alpha)-x_{2} \cos (\alpha)\right)^{2} \mathrm{~d} \alpha \\
& =\frac{1}{\beta}\left[x_{1}^{2}\left(\frac{\beta-\sin (\beta) \cos (\beta)}{2}\right)+x_{2}^{2}\left(\frac{\beta+\sin (\beta) \cos (\beta)}{2}\right)-x_{1} x_{2} \sin ^{2}(\beta)\right] .
\end{aligned}
$$


Using that for $x=\lambda e_{\alpha}$ with $\lambda \geq 0$ holds $\operatorname{dist}(x, C)=\lambda$ and $R_{\beta}(x)=\lambda^{2} R_{\beta}\left(e_{\alpha}\right)$ and employing trigonomertric calculation rules, we find the regularity constant in Eq. (14) not to be smaller than

$$
\kappa=\sup _{x \in \mathbb{R}^{2}} \frac{\operatorname{dist}^{2}(x, C)}{R_{\beta}(x)}=\sup _{\alpha \in[0,2 \pi)} \frac{8 \beta}{2 \beta-\sin (2 \beta-2 \alpha)-\sin (2 \alpha)}=\frac{4 \beta}{\beta-\sin (\beta)},
$$

where the last supremum is attained at $\alpha=\frac{\beta}{2}$. So from Theorem 3.11 we get uniform geometric convergence.

Example 4.9 (disks on a circle). This example illustrates Theorem 4.3. Let $C_{\alpha}:=\overline{\mathbb{B}}\left(\rho e_{\alpha}, 1\right) \subset$ $\mathbb{R}^{2}$, where $0<\rho<1$ and $e_{\alpha}=\left(\begin{array}{c}\cos (\alpha) \\ \sin (\alpha)\end{array}\right), \alpha \in[0,2 \pi)$ and let $\xi \sim$ unif $[0,2 \pi]$.

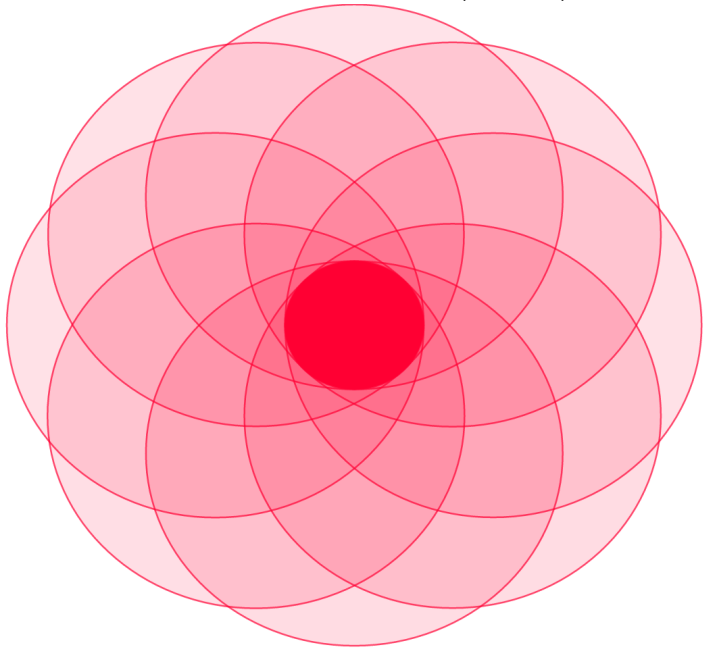

Figure 3

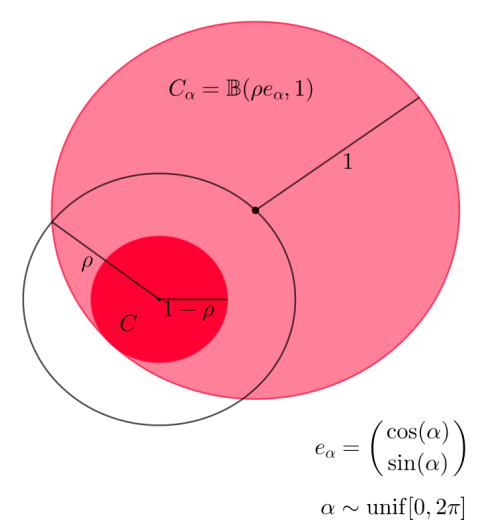

Figure 4

The intersection is given by $C=\overline{\mathbb{B}}(0,1-\rho)$. We show next that sets with this configuration do not satisfy (14). To see this we show that there is a sequence $\left(x_{n}\right)_{n} \subset \mathbb{R}^{2}$ with $\mathbb{P}\left(x_{n} \in C_{\xi}\right) \rightarrow 1$ as $n \rightarrow \infty$. By Theorem 4.3 we conclude that (14) cannot hold. Indeed, let $x=x(\lambda)=\lambda\left(\begin{array}{l}1 \\ 0\end{array}\right)$ with $\lambda \geq 1-\rho$, then

$$
\begin{aligned}
\mathbb{P}\left(x \in C_{\xi}\right) & =\frac{1}{2 \pi} \int_{0}^{2 \pi} \mathbb{1}\left\{\left\|x-\rho e_{\alpha}\right\| \leq 1\right\} \mathrm{d} \alpha \\
& =\frac{1}{2 \pi} \int_{0}^{2 \pi} \mathbb{1}\left\{\lambda^{2}+\rho^{2}-2 \lambda \rho \cos (\alpha) \leq 1\right\} \mathrm{d} \alpha \\
& =\frac{1}{2 \pi} \int_{-\beta}^{\beta} 1 \mathrm{~d} \alpha \\
& =\frac{\beta}{\pi},
\end{aligned}
$$

where $\beta=\beta(\lambda)=\cos ^{-1}\left(\frac{\lambda^{2}+\rho^{2}-1}{2 \lambda \rho}\right)$, if $\lambda \leq 1+\rho$. We have $\beta(\lambda) \rightarrow \pi$ as $\lambda \rightarrow 1-\rho$, so $\mathbb{P}\left(x(\lambda) \in C_{\xi}\right) \rightarrow 1$ as $\lambda \rightarrow 1-\rho$.

In contrast to the case where $\rho \in(0,1)$, the extreme cases where $\rho=0$ and $\rho=1$ do satisfy (14). This will not be shown here.

\subsection{RFI with two families of mappings}

The set feasibility examples above lead very naturally to the more general context of mappings $T_{i}: G \rightarrow G, i \in I$ and $S_{j}: G \rightarrow G, j \in J$ on a metric space $(G, d)$, where $I, J$ are 
arbitrary index sets. Here we envision the scenario where $C_{T}:=\left\{x \in G \mid \mathbb{P}\left(x \in\right.\right.$ Fix $\left.\left.T_{\xi}\right)=1\right\}$ and $C_{S}:=\left\{x \in G \mid \mathbb{P}\left(x \in\right.\right.$ Fix $\left.\left.S_{\zeta}\right)=1\right\}$ are distinctly different sets, possibly nonintersecting. Let $(\Omega, \mathcal{F}, \mathbb{P})$ be a probability space and let $\xi: \Omega \rightarrow I, \zeta: \Omega \rightarrow J$ be two random variables. Let $\left(\xi_{n}\right)_{n \in \mathbb{N}}$ be an iid sequence with $\xi_{n} \stackrel{\mathrm{d}}{=} \xi$ and $\left(\zeta_{n}\right)_{n \in \mathbb{N}}$ iid with $\zeta_{n} \stackrel{\mathrm{d}}{=} \zeta$. The two sequences are assumed to be independent of each other. Let $\mu$ be a probability measure on $(G, \mathcal{B}(G))$. Consider the stochastic selection method for two families of mappings

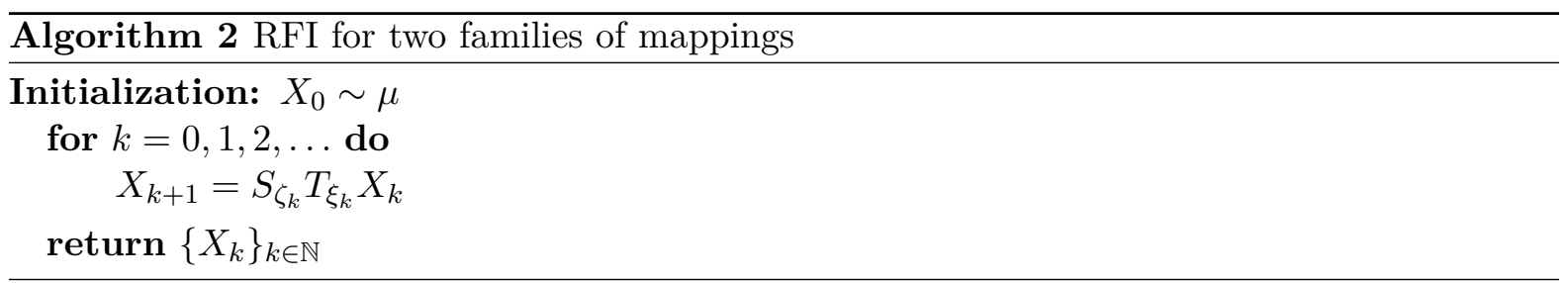

Note, that this structure of two families of mappings is a special case of Algorithm 1, just set $\tilde{T}_{(i, j)}=S_{j} T_{i}$, where $(i, j) \in \tilde{I}=I \times J$ and $\tilde{\xi}=(\xi, \zeta)$. Also the Markov chain property is still satisfied, the transition kernel takes the form $p(x, A)=\mathbb{P}\left(S_{\zeta} T_{\xi} x \in A\right)$ for $x \in G$ and $A \in \mathcal{B}(G)$. An advantage of this formulation is that properties of the two families $\left\{S_{j}\right\}_{j \in J}$ and $\left\{T_{i}\right\}_{i \in I}$ can be analyzed more specifically, and independently. As long as the mapping $\tilde{T}$ satisfies the properties needed for convergence of the RFI, then convergence of the RFI for two families of mappings follows. At the very least, we need

$$
C:=\left\{x \in G \mid \mathbb{P}\left(x \in \operatorname{Fix} \tilde{T}_{\tilde{\xi}}\right)=1\right\} \neq \emptyset .
$$

From this it is easy to see that for convergence the set $C_{T}$ could be empty, but the set $C_{S}$ must be nonempty.

Example 4.10 (consistent feasibility). Revisit Example 4.6. We had $C_{1}=\mathbb{R}_{+} \times \mathbb{R}$ and $C_{2}=$ $\mathbb{R} \times \mathbb{R}_{+}$. Set $I=\{1\}$ and $J=\{2\}$, then the algorithm is the deterministic alternating projections method. One has $\mathbb{P}\left(X_{1} \in C\right)=1$ for all initial distributions.

Example 4.11 (inconsistent stochastic feasibility). In this example we show that the framework established here is not exclusively limited to consistent feasibility. Consider the (trivially convex, nonempty and closed) set $S:=\{(0,10)\}$ together with the collection of balls in Example 4.9, $C_{\alpha}:=\overline{\mathbb{B}}\left(\rho e_{\alpha}, 1\right) \subset \mathbb{R}^{2}$, where $0 \leq \rho \leq 1$ and $e_{\alpha}=\left(\begin{array}{c}\cos (\alpha) \\ \sin (\alpha)\end{array}\right), \alpha \in[0,2 \pi)$ and let $\xi \sim$ unif $[0,2 \pi]$. The intersection of the disks is given by $C_{T}=\overline{\mathbb{B}}(0,1-\rho)$ where $T_{\alpha}:=P_{C_{\alpha}}$ is the metric projection onto $C_{\alpha}$. Although $S \cap C_{\alpha}=\emptyset$ for all $\alpha \in[0,2 \pi)$, still the fixed point set for the mapping in Algorithm 2 (where $S_{\zeta}=P_{S}$ ) is $C=\{(0,10)\}$, and this is found after one iteration for any initial probability distribution $\mu$, where $X_{0} \sim \mu$.

This is indeed a special example, but points to the richness of inconsistent stochastic feasibility, which will be studied in greater depth in a follow-up paper.

\subsection{Linear Operator equations}

There are several applications of the RFI to the feasibility problem [1], [4]. We want to focus first on linear operator equations in the separable Hilbert space $\mathcal{H}=L_{2}([a, b])$. Let $T: \mathcal{H} \rightarrow \mathcal{H}$ be a bounded linear operator, we want to find $x \in \mathcal{H}$, such that

$$
T x=g,
$$

for a given $g \in \mathcal{H}$. Clearly this is possible only if $g \in R(T)$. The idea in [4] to solve $T x=g$ is to consider the family of evaluation mappings $\varphi_{t}: \mathcal{H} \rightarrow \mathbb{R}, t \in[a, b]$, which are given by

$$
\varphi_{t}(x):=(T x)(t) .
$$


Define the affine subspaces $C_{t}:=\left\{x \in \mathcal{H} \mid \varphi_{t}(x)=g(t)\right\}, t \in[a, b]$. Consider the probability space $(\Omega, \mathcal{F}, \mathbb{P})=\left([a, b], \mathcal{B}([a, b]), \frac{\lambda}{b-a}\right)$, where $\lambda$ is the Lebesgue-measure. Let $\xi:(\Omega, \mathcal{F}, \mathbb{P}) \rightarrow$ $([a, b], \mathcal{B}([a, b]))$ be a random variable with $\mathbb{P}^{\xi}=\mathbb{P}=\frac{\lambda}{b-a}$. Then for $g \in R(T)$, we have that

$$
T x=g \quad \text { if and only if } \quad x \in C:=\left\{y \in \mathcal{H} \mid \mathbb{P}\left(y \in C_{\xi}\right)=1\right\} .
$$

So the linear operator equation becomes a stochastic feasibility problem.

In order to be able to compute projections onto the sets $C_{t}, t \in[a, b]$, we need the evaluation functionals $\varphi_{t}$ to be continuous, i.e. $\left\|\varphi_{t}\right\|<\infty$ for almost all $t \in[a, b]$. By the Riesz representation theorem there exists a unique $u_{t} \in \mathcal{H}$ with $\varphi_{t}(x)=\left\langle u_{t}, x\right\rangle$ for all $x \in \mathcal{H}$ and almost all $t \in[a, b]$. We conclude that the projection onto $C_{t}$ takes the form

$$
P_{t} x=x+\frac{g(t)-(T x)(t)}{\left\|u_{t}\right\|^{2}} u_{t} \quad x \in L_{2}([a, b]) .
$$

Example 4.12 (linear integral equations). Concretely, consider an integral equation of the first kind in the separable Hilbert space $L_{2}([a, b])$

$$
(T x)(t)=\int_{a}^{b} K(t, s) x(s) \mathrm{d} s=g(t) \quad t \in[a, b],
$$

with $g \in L_{2}([a, b])$. For $K \in L_{2}([a, b] \times[a, b]), T$ is a continuous linear compact operator [18, Theorem 8.15] (a Hilbert-Schmidt operator). For the Riesz representation of the evaluation functionals we have that $\varphi_{t}(x)=(T x)(t)=\left\langle u_{t}, x\right\rangle, t \in[a, b]$, as well as $u_{t}=K(t, \cdot)$ and hence $\left\|\varphi_{t}\right\| \leq\|K(t, \cdot)\|<\infty$.

Example 4.13 (differentiation). Let $K(t, s)=\mathbb{1}_{[a, t]}(s)=u_{t}(s)$, i.e. $(T x)(t)=\int_{a}^{t} x(s) \mathrm{d} s$ and suppose $g \in C^{1}([a, b])$, then $T x=g$ if and only if $x=g^{\prime}$ almost surely and $g(a)=0$. The projectors take the form

$$
P_{t} x=x-\frac{g(t)-\int_{a}^{t} x(s) \mathrm{d} s}{t-a} \mathbb{1}_{[a, t]} .
$$

\section{A. Appendix}

Lemma A.1 (slices of product $\sigma$-field, see Proposition 3.3.2 in [19]). Let $\left(\Omega_{i}, \mathcal{F}_{i}\right), i=1,2$ be two measurable spaces and $M \in \mathcal{F}_{1} \otimes \mathcal{F}_{2}$. Then for $\omega_{1} \in \Omega_{1}$ holds $M_{\omega_{1}}:=\left\{\omega_{2} \in \Omega_{2} \mid\left(\omega_{1}, \omega_{2}\right) \in M\right\} \in$ $\mathcal{F}_{2}$.

Theorem A.2 (dense sets in separable metric space). Let $(G, d)$ be a Polish space (complete separable metric space). Then for any $A \subset G$, there is a dense countable subset $\left\{a_{n}\right\}_{n \in \mathbb{N}} \subset A$ and if $A$ is closed then even $A=\operatorname{cl}\left\{a_{n}\right\}$ (cl $U$ denotes the closure of the set $U \subset G$ w.r.t. the metric d).

Proof. Since $G$ is separable there exists a dense and countable subset $\left\{u_{n}\right\}_{n \in \mathbb{N}} \subset G$ with $G=$ $\operatorname{cl}\left\{u_{n}\right\}_{n}$. By denseness of $\left\{u_{n}\right\} \subset G$, for any $x \in G$ and any $\epsilon>0$, there is $u_{n}$, where $n$ is depending on $x$ and $\epsilon$, with $d\left(u_{n}, x\right)<\epsilon$. Let $\epsilon>0$ and choose $a_{n}^{\epsilon} \in \mathbb{B}\left(u_{n}, \epsilon\right) \cap A, n \in \mathbb{N}$, if the intersection is nonempty. The set $\tilde{A}:=\left\{a_{n}^{1 / m}\right\}_{n, m \in \mathbb{N}} \subset A$ is nonempty and countable as union of countable sets. It holds for any $a \in A$ and any $\epsilon>0$ that $\exists n, m$ with $1 / m<\epsilon$ and $d\left(a, u_{n}\right)<\epsilon$, hence

$$
d\left(a, a_{n}^{1 / m}\right) \leq d\left(a, u_{n}\right)+d\left(u_{n}, a_{n}^{1 / m}\right)<2 \epsilon,
$$

i.e. $\tilde{A} \subset A$ dense. So then $A \subset \operatorname{cl} \tilde{A}$ and if $A$ is closed, then also $\operatorname{cl} \tilde{A} \subset A$. 
Theorem A.3 (support of a measure). Let $(G, d)$ be a Polish space and $\mathcal{B}(G)$ its Borel $\sigma$ algebra. Let $\pi$ be a measure on $(G, \mathcal{B}(G))$ and define its support via

$$
\operatorname{supp} \pi=\{x \in G \mid \pi(\mathbb{B}(x, \epsilon))>0 \forall \epsilon>0\} .
$$

Then

1. $\operatorname{supp} \pi \neq \emptyset$, if $\pi \neq 0$.

2. $\operatorname{supp} \pi$ is closed.

3. $\pi(A)=\pi(A \cap \operatorname{supp} \pi)$ for all $A \in \mathcal{B}(G)$, i.e. $\pi\left((\operatorname{supp} \pi)^{c}\right)=0$.

4. For any closed set $S \subset G$ with $\pi(A \cap S)=\pi(A)$ for all $A \in \mathcal{B}(G)$ holds $\operatorname{supp} \pi \subset S$.

Proof. 1. If $\pi(G)>0$, then due to separability one could find for any $\epsilon_{1}>0$ a countable cover of $G$ with balls with radius $\epsilon_{1}$, where at least one needs to have nonzero measure, because $0<\pi(G) \leq \sum_{n} \pi\left(\mathbb{B}\left(x_{n}, \epsilon\right)\right)$. Now just consider $B_{1}:=\mathbb{B}\left(x_{N}, \epsilon_{1}\right)$ such that $\pi\left(B_{1}\right)>0$ and apply the above procedure of countable covers with $\epsilon_{2}<\epsilon_{1}$ iteratively, then there is a sequence $\epsilon_{n} \rightarrow 0$ and $\mathbb{B}\left(x_{n+1}, \epsilon_{n+1}\right) \subset \mathbb{B}\left(x_{n}, \epsilon_{n}\right)$, such that $x_{n} \rightarrow x$, i.e. $x \in \operatorname{supp} \pi$.

2. Let $\left(x_{n}\right)_{n \in \mathbb{N}} \subset \operatorname{supp} \pi$ with $x_{n} \rightarrow x$ as $n \rightarrow \infty$. Let $\epsilon>0$ and $N>0$ such that $d\left(x_{n}, x\right)<\epsilon$ for all $n \geq N$. Then $x_{n} \in \mathbb{B}(x, \epsilon)$ and $\exists \tilde{\epsilon}>0$ with $\mathbb{B}\left(x_{n}, \tilde{\epsilon}\right) \subset \mathbb{B}(x, \epsilon)$, so we get

$$
\pi(\mathbb{B}(x, \epsilon)) \geq \pi\left(\mathbb{B}\left(x_{n}, \tilde{\epsilon}\right)\right)>0,
$$

i.e. $x \in \operatorname{supp} \pi$.

3. Write $S=(\operatorname{supp} \pi)^{c}$. Choose $\left\{x_{n}\right\}_{n \in \mathbb{N}} \subset S$ dense. By openness of $S$ there exists $\epsilon_{n}>0$ with $\mathbb{B}\left(x_{n}, \epsilon_{n}\right) \subset S$, hence $S=\bigcup_{n \in \mathbb{N}} \mathbb{B}\left(x_{n}, \epsilon_{n}\right)$ and

$$
\pi(S) \leq \sum_{i \in \mathbb{N}} \pi\left(\mathbb{B}\left(x_{n}, \epsilon_{n}\right)\right)=0 .
$$

(It holds $\pi\left(\mathbb{B}\left(x_{n}, \epsilon_{n}\right)\right)=0$, because otherwise, one could find for any small enough $\epsilon>0$ a countable cover of $\mathbb{B}\left(x_{n}, \epsilon_{n}\right)$ with balls with radius $\epsilon$, where at least one needs to have nonzero measure. Since this holds for all $\epsilon$, there is a contradiction to $\mathbb{B}\left(x_{n}, \epsilon_{n}\right) \subset S$.)

4. Let $x \in \operatorname{supp} \pi$. So $\pi(\mathbb{B}(x, \epsilon) \cap S)>0$ for all $\epsilon>0$, i.e. $\mathbb{B}(x, \epsilon) \cap S \neq \emptyset$ for all $\epsilon>0$. Let $x_{n}$ be such that $x_{n} \in \mathbb{B}\left(x, \epsilon_{n}\right) \cap S$, where $\epsilon_{n} \rightarrow 0$ as $n \rightarrow \infty$. Then by closedness of $S$, $x_{n} \rightarrow x \in S$.

Theorem A.4 (construction of an invariant measure). Let $\left(\mu \mathcal{P}^{n}\right)_{n \in \mathbb{N}}$ be a tight family of probability measures on a Polish space $(G, d)$, i.e. for any $\epsilon>0$ there exists $K_{\epsilon} \subset G$ compact with $\left(\mu \mathcal{P}^{n}\right)\left(G \backslash K_{\epsilon}\right)<\epsilon$ for all $n \in \mathbb{N}$, where $\mu \in \mathscr{P}(G)$ and $\mathcal{P}$ is the Markov operator for a given transition kernel $p$, which is assumed to be Feller. Then any clusterpoint of $\left(\nu_{n}\right)$ where $\nu_{n}=\frac{1}{n} \sum_{i=1}^{n} \mu \mathcal{P}^{i}$ is an invariant measure for $\mathcal{P}$.

Proof. This is basically [20, Theorem 1.10]. The tightness of $\left(\mu \mathcal{P}^{n}\right)$ implies tightness of $\left(\nu_{n}\right)$ and therefore there exists a weakly converging subsequence $\left(\nu_{n_{k}}\right)$ with limit $\pi \in \mathscr{P}(G)$ by Prokhorov's Theorem. By the Feller property of $\mathcal{P}$ one has for any continuous and bounded $f: G \rightarrow \mathbb{R}$ that also $\mathcal{P} f$ is continuous and bounded and hence

$$
|(\pi \mathcal{P}) f-\pi f|=|\pi(\mathcal{P} f)-\pi f|
$$




$$
\begin{aligned}
& =\lim _{k}\left|\nu_{n_{k}}(\mathcal{P} f)-\nu_{n_{k}} f\right| \\
& =\lim _{k} \frac{1}{n_{k}}\left|\mu \mathcal{P}^{n_{k}+1} f-\mu \mathcal{P} f\right| \\
& \leq \lim _{k} \frac{2\|f\|_{\infty}}{n_{k}} \\
& =0 .
\end{aligned}
$$

Theorem A.5 (conditional expectation - basics, see Theorem 5.1 in [7]). Let $(\Omega, \mathcal{F}, \mathbb{P})$ be a probability space and $X$ a real-valued random variable with $\mathbb{E}|X|<\infty$ (integrable). Let $\mathcal{F}_{0} \subset \mathcal{F}$ a sub- $\sigma$-algebra. Then it exists an a.s. unique $\mathcal{F}_{0}-m b$. random variable $Z:=\mathbb{E}\left[X \mid \mathcal{F}_{0}\right]$ with $\mathbb{E}\left(Z \mathbb{1}_{A}\right)=\mathbb{E}\left(X \mathbb{1}_{A}\right)$ for all $A \in \mathcal{F}_{0}$.

Let $Y, X_{n}$ be integrable random variables. Further properties are:

1. $\mathbb{E}\left(\mathbb{E}\left[X \mid \mathcal{F}_{0}\right]\right)=\mathbb{E} X$.

2. $X$ is $\mathcal{F}_{0}-m b$, then $\mathbb{E}\left[X \mid \mathcal{F}_{0}\right]=X$ a.s.

3. $X$ independent of $\mathcal{F}_{0}$, then $\mathbb{E}\left[X \mid \mathcal{F}_{0}\right]=\mathbb{E} X$ a.s.

4. $\mathbb{E}\left[a X+b Y \mid \mathcal{F}_{0}\right]=a \mathbb{E}\left[X \mid \mathcal{F}_{0}\right]+b \mathbb{E}\left[Y \mid \mathcal{F}_{0}\right]$ a.s. for all $a, b \in \mathbb{R}$.

5. $X \leq Y$, then $\mathbb{E}\left[X \mid \mathcal{F}_{0}\right] \leq \mathbb{E}\left[Y \mid \mathcal{F}_{0}\right]$ a.s.

6. $0 \leq X_{n} \uparrow X$, then $\mathbb{E}\left[X_{n} \mid \mathcal{F}_{0}\right] \uparrow \mathbb{E}\left[X \mid \mathcal{F}_{0}\right]$ a.s.

7. $\mathcal{F}_{0} \subset \mathcal{F}_{1} \subset \mathcal{F}$ with $\sigma$-algebra $\mathcal{F}_{1}$, then $\mathbb{E}\left[\mathbb{E}\left[X \mid \mathcal{F}_{1}\right] \mid \mathcal{F}_{0}\right]=\mathbb{E}\left[X \mid \mathcal{F}_{0}\right]$.

Lemma A.6 (Satz 17.11 in [21]). Let $(\Omega, \mathcal{F})$ be a measurable space and $\mu$ be $\sigma$-finite. Let $f: \Omega \rightarrow[0, \infty]$ and set $\nu=f \cdot \mu$ (i.e. $\nu(A)=\int_{A} f \mathrm{~d} \mu$ for $\left.A \in \mathcal{F}\right)$. Then $f$ is $\mu$-a.s. unique. Furthermore, $\nu$ is $\sigma$-finite (i.e. there exists $\left(\Omega_{n}\right)_{n \in \mathbb{N}} \subset \mathcal{F}$ with $\nu\left(\Omega_{n}\right)<\infty$ and $\Omega_{n} \uparrow \Omega$ ) if and only if $f$ is real-valued $\mu$-a.s.

Remark A.7: A nonnegative real-valued random variable $X$ on a probability space $(\Omega, \mathcal{F}, \mathbb{P})$ induces a $\sigma$-finite measure $\nu=X \cdot \mathbb{P}$.

Theorem A.8 (conditional expectation - extension). Let $(\Omega, \mathcal{F}, \mathbb{P})$ be a probability space and $X \geq 0$ be a real-valued random variable. Let $\mathcal{F}_{0} \subset \mathcal{F}$ be a sub- $\sigma$-algebra. Then it exists an a.s. unique nonnegative real-valued random variable $Z:=\mathbb{E}\left[X \mid \mathcal{F}_{0}\right]$ on $\left(\Omega, \mathcal{F}_{0}\right)$ with $\mathbb{E}\left(Z \mathbb{1}_{A}\right)=$ $\mathbb{E}\left(X \mathbb{1}_{A}\right)$ for all $A \in \mathcal{F}_{0}$.

Let additionally $Y,\left(X_{n}\right)$ be nonnegative and real-valued, then all items 1.-7. in Theorem A.5 are satisfied for these.

Proof. From Remark A.7 follows the existence of disjoint sets $\Omega_{n} \in \mathcal{F}_{0}$ with $\bigcup_{n} \Omega_{n}=\Omega$ and the property that $\int_{\Omega_{n}} X \mathrm{~d} \mathbb{P}<\infty$. One has that a.s.

$$
\mathbb{1}_{\Omega_{n}} \mathbb{E}\left[X \mid \mathcal{F}_{0} \cap \Omega_{n}\right]=\mathbb{E}\left[X \mathbb{1}_{\Omega_{n}} \mid \mathcal{F}_{0} \cap \Omega_{n}\right]=\mathbb{E}\left[X \mid \mathcal{F}_{0} \cap \Omega_{n}\right]=\mathbb{E}\left[X \mathbb{1}_{\Omega_{n}} \mid \mathbb{F}_{0}\right] .
$$

Define $Z:=\sum_{n} \mathbb{E}\left[X \mid \mathcal{F}_{0} \cap \Omega_{n}\right]$, then $Z=\mathbb{E}\left[X \mid \mathcal{F}_{0}\right]$. The items 1.-7. follow now from Theorem A.5 on $\Omega_{n}$ and the Monotone Convergence Theorem. 
Theorem A.9 (disintegration). Let $(\Omega, \mathcal{F}, \mathbb{P})$ be a probability space and $\left(\Omega_{1}, \mathcal{F}_{1}\right),\left(\Omega_{2}, \mathcal{F}_{2}\right)$ be measurable spaces and $\mathcal{F}_{0} \subset \mathcal{F}$ a sub- $\sigma$-algebra, let $X_{1} \in \Omega_{1}$ have a regular version $\mu$ of $\mathbb{P}\left(X_{1} \in \cdot \mid \mathcal{F}_{0}\right)$ and let $X_{2} \in \Omega_{2}$ be $\mathcal{F}_{0}-m b$. Let furthermore $f: \Omega_{1} \times \Omega_{2} \rightarrow \mathbb{R}_{+}$be measurable. Then

$$
\mathbb{E}\left[f\left(X_{1}, X_{2}\right) \mid \mathcal{F}_{0}\right]=\int f\left(x_{1}, X_{2}\right) \mu\left(\mathrm{d} x_{1}\right) \quad \text { a.s. }
$$

Lemma A.10. Let $(\Omega, \mathcal{F}, \mathbb{P})$ be probability space. Let $\left(X_{k}\right)_{k \in \mathbb{N}_{0}},\left(U_{k}\right)_{k \in \mathbb{N}_{0}}$ be sequences of nonnegative real-valued random variables with $X_{k} \in \mathcal{F}_{k}$, where $\mathcal{F}_{0} \subset \mathcal{F}_{1} \subset \ldots \subset \mathcal{F}$ are $\sigma$ algebras. Suppose for all $k \in \mathbb{N}_{0}$

$$
X_{k+1} \leq X_{k}-U_{k} \quad \text { a.s. }
$$

Define $V_{k}:=\mathbb{E}\left[U_{k} \mid \mathcal{F}_{k}\right]$ for $k \in \mathbb{N}_{0}$. Then $X_{k} \rightarrow X$ a.s. and $\sum_{k} U_{k}, \sum_{k} V_{k}<\infty$ a.s.

Proof. This is a special instance of the more general supermartingale convergence theorem in [22].

Lemma A.11 (further properties of $R$ ). Under the standing assumptions, if $T_{i}=P_{i}$ are projectors onto nonempty, closed and convex sets, $i \in I$. There holds that:

1. $R$ is convex.

2. $R$ is continuously differentiable, $\frac{1}{2} \nabla R(x)=x-\mathbb{E}\left[P_{\xi} x\right]$ for all $x \in \mathcal{H}$.

3. $\nabla R$ is globally Lipschitz continuous with constant not larger than 4 .

4. $C=\{\nabla R=0\}$.

Proof. 1. The function $x \mapsto \operatorname{dist}\left(x, C_{i}\right)$ is convex for all $i \in I$, since $C_{i}=$ Fix $P_{i}$ is convex, nonempty and closed. On $[0, \infty)$ the function $x \mapsto x^{2}$ is increasing and convex, so $x \mapsto$ $\operatorname{dist}^{2}\left(x, C_{i}\right)$ is convex, $i \in I$. The convexity of $R$ follows by linearity of the expectation.

2. We need to show that

$$
\lim _{0 \neq\|y\| \rightarrow 0} \frac{\left|R(x+y)-R(x)-2 \mathbb{E}\left[\left\langle x-P_{\xi} x, y\right\rangle\right]\right|}{\|y\|}=0 .
$$

Let $\left(y_{n}\right) \subset \overline{\mathbb{B}}(0, \epsilon) \subset \mathcal{H}$ with $y_{n} \rightarrow 0$. Define a sequence of functions on $\Omega$ via

$$
f_{n}=\frac{\left|\operatorname{dist}^{2}\left(x+y_{n}, C_{\xi}\right)-\operatorname{dist}^{2}\left(x, C_{\xi}\right)-2\left\langle x-P_{\xi} x, y_{n}\right\rangle\right|}{\left\|y_{n}\right\|} .
$$

Then for fixed $\omega \in \Omega$ we have $f_{n}(\omega) \rightarrow 0$ as $n \rightarrow \infty$ since the function $x \mapsto \operatorname{dist}^{2}\left(x, C_{i}\right)$ is Fréchet differentiable for all $i \in I$ [8, Corollary 12.30]. Furthermore, we find for any $n \in \mathbb{N}$ that

$$
\begin{aligned}
f_{n} & =\frac{\left|\left\|x+y_{n}-P_{\xi}\left(x+y_{n}\right)\right\|^{2}-\left\|x-P_{\xi} x\right\|^{2}-2\left\langle x-P_{\xi} x, y_{n}\right\rangle\right|}{\left\|y_{n}\right\|} \\
& =\frac{\left|\left\|y_{n}-P_{\xi}\left(x+y_{n}\right)+P_{\xi} x\right\|^{2}+2\left\langle x-P_{\xi} x, P_{\xi} x-P_{\xi}\left(x+y_{n}\right)\right\rangle\right|}{\left\|y_{n}\right\|}
\end{aligned}
$$




$$
\begin{aligned}
& =\frac{\left|\left\|y_{n}\right\|^{2}+\left\|P_{\xi} x-P_{\xi}\left(x+y_{n}\right)\right\|^{2}+2\left\langle y_{n}+x-P_{\xi} x, P_{\xi} x-P_{\xi}\left(x+y_{n}\right)\right\rangle\right|}{\left\|y_{n}\right\|} \\
& \leq \frac{4\left\|y_{n}\right\|^{2}+2 \operatorname{dist}\left(x, C_{\xi}\right)\left\|y_{n}\right\|}{\left\|y_{n}\right\|} \\
& \leq 4 \epsilon+2 \operatorname{dist}\left(x, C_{\xi}\right)=: g,
\end{aligned}
$$

where, in the first inequality, we used nonexpansivity of the projectors $P_{i}, i \in I$ and the Cauchy-Schwartz inequality. In particular with Hölder's inequality follows that $\mathbb{E}[g] \leq$ $4 \epsilon+2 \sqrt{R(x)}$, i.e. $g$ is integrable and hence Lebesgue's Dominated Convergence Theorem yields $\mathbb{E}\left[f_{n}\right] \rightarrow 0$, which gives us Fréchet differentiability of $R$ with derivative $\nabla R(x)=$ $2 \mathbb{E}\left[x-P_{\xi} x\right]$. Continuity of $\nabla R$ follows from

$$
\begin{aligned}
\|\nabla R(x+y)-\nabla R(x)\| & =2\left\|\mathbb{E}\left[y-P_{\xi}(x+y)+P_{\xi} x\right]\right\| \\
& \leq 2 \mathbb{E}\left[\|y\|+\left\|P_{\xi}(x+y)-P_{\xi} x\right\|\right] \\
& \leq 4\|y\|,
\end{aligned}
$$

where we used nonexpansivity of the projectors $P_{i}, i \in I$ in the second inequality.

3. For any $x, y \in \mathcal{H}$ it holds that $\|\nabla R(x)-\nabla R(y)\| \leq 2\left(\|x-y\|+\left\|\mathbb{E}\left[P_{\xi} x-P_{\xi} y\right]\right\|\right)$. Applying Jensen's inequality and nonexpansivity, we arrive at the desired result.

4. Clearly if $x \in C$, then $x=P_{\xi} x$ a.s. and so $x=\mathbb{E}\left[P_{\xi} x\right]$, i.e. $\nabla R(x)=0$ by 2 .

Now conversely, if $\nabla R(x)=0$, then by convexity $R(x)-R(y) \leq\langle\nabla R(x), x-y\rangle=0$ for all $y \in \mathcal{H}$. Since $C \neq \emptyset$ there is $y \in \mathcal{H}$ with $R(y)=0$, so also $R(x)=0$, i.e. $x \in C$.

\section{B. Paracontractions}

Paracontractions include the set of averaged operators, but averaged mappings possess more useful regularity properties, e.g. when composing these operators, one stays in the set of averaged operators, whereas for nonaveraged operators this is not clear in general. An example of a nonaveraged paracontraction in $\mathbb{R}$ is a Huber function with parameter $\alpha>0$ (see also [23, Example 2.3] for $\alpha=1$ )

$$
f_{\alpha}(x):=\left\{\begin{array}{ll}
\frac{x^{2}}{2 \alpha}, & |x| \leq \alpha \\
|x|-\frac{\alpha}{2}, & |x|>\alpha
\end{array}, \quad x \in \mathbb{R} .\right.
$$

We have that $f_{\alpha}$ is nonexpansive and paracontractive, but not averaged, since for $x=-2 \alpha$ and $y=-\alpha$ one has $f(x)=\frac{3 \alpha}{2}$ and $f(y)=\frac{\alpha}{2}$. Consequently

$$
|f(x)-f(y)|=\alpha=|x-y|, \quad \text { but } \quad|x-f(x)-(y-f(y))|=2 \alpha \neq 0 .
$$

In general metric spaces with nonlinear structure the averaged mappings are not defined or at least demand a different definition, but still the paracontraction framework applies here and exhibits a useful description of mappings which ensure that the RFI converges to a common fixed point. Paracontractions were used in Section 3.1 to guarantee Fejér monotonicity, yielding convergence; averagedness in this context would be too strong an assumption (and is not defined actually). In the following we provide an example of a class of paracontracting operators in $\mathbb{R}^{n}$, that are not in general averaged, resolvents of quasiconvex functions. 
Definition B.1. A function $f: \mathbb{R}^{n} \rightarrow \mathbb{R}$ is called quasiconvex, if the sublevel sets

$$
\left\{x \in \mathbb{R}^{n} \mid f(x) \leq \alpha\right\}
$$

are convex for all $\alpha \in \mathbb{R}$. Equivalently, $f$ satisfies

$$
f(\lambda x+(1-\lambda) y) \leq \max \{f(x), f(y)\} \quad \forall x, y \in \mathbb{R}^{n}, \forall \lambda \in[0,1] .
$$

The proximity operator function $f: \mathbb{R}^{n} \rightarrow \mathbb{R}$ is given by the set-valued mapping

$$
\operatorname{prox}_{f}(x):=\underset{y \in \mathbb{R}^{n}}{\operatorname{argmin}}\left\{f(y)+\frac{1}{2}\|x-y\|^{2}\right\}, \quad x \in \mathbb{R}^{n} .
$$

Lemma B.2. Let $f: \mathbb{R}^{n} \rightarrow \mathbb{R}$ be twice differentiable and quasiconvex and satisfy $S:=$ $\operatorname{argmin} f \neq \emptyset$ and $\nabla f \neq 0$ on $\mathbb{R}^{n} \backslash S$, furthermore suppose that $\operatorname{Id}+\operatorname{Hess} f(x)$ is positive definit for all $x \in \mathbb{R}^{n}$, then $\operatorname{prox}_{f}$ is paracontracting.

Proof. Denote $A:=\operatorname{Id}+\nabla f$. Let $x, y \in \mathbb{R}^{n}$ with $f(x) \geq f(y)$, then

$$
\|A(x)-y\|^{2}=\|x-y\|^{2}+\|\nabla f(x)\|^{2}+\langle\nabla f(x), x-y\rangle \geq\|x-y\|^{2},
$$

where we used that in [24] it is shown, that a quasiconvex and differentiable function satisfies

$$
f(x) \geq f(y) \quad \Longrightarrow \quad\langle\nabla f(x), x-y\rangle \geq 0,
$$

for any $x, y \in \mathbb{R}^{n}$. Note that if $x \notin S$ then $\nabla f(x) \neq 0$ by assumption and hence for $y \in \mathbb{R}^{n}$ with $f(y) \leq f(x)$ it holds that

$$
\|A(x)-y\|>\|x-y\| .
$$

Moreover, the function

$$
g(y):=f(y)+\frac{1}{2}\|x-y\|^{2}
$$

for fixed $x \in \mathbb{R}^{n}$ is bounded from below, since $\inf _{x} f(x)>-\infty$ by assumption and coercive. From positive definitness of Id + Hess $f$ we have that $g$ is also twice continuously differentiable and strictly convex, hence it possesses a unique minimizer $\bar{x}$ that satisfies

$$
x=\nabla f(\bar{x})+\bar{x}=A(\bar{x}),
$$

it follows that $A\left(\mathbb{R}^{n}\right)=\mathbb{R}^{n}$, i.e. $A$ is surjective. Furthermore, $A$ is injective, since from uniqueness of the minimizer and sufficiency of the first order optimality criterion for $\bar{x}$ to be a minimizer ( $g$ is convex) it follows that, if $A(\bar{x})=A(\bar{y})$, then $\bar{x}=\bar{y}$ is the minimizer for $g$ and in particular $A(\bar{x})=x \Leftrightarrow \operatorname{prox}_{f}(x)=\bar{x}$.

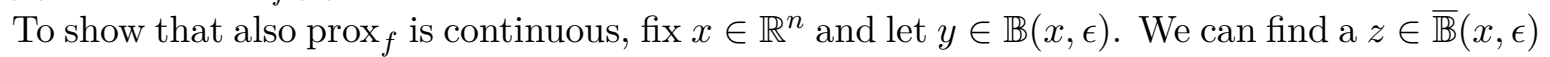
with $f(z) \leq f(y)$ for all $y \in \overline{\mathbb{B}}(x, \epsilon)$ by continuity of $f$, so we get with (19) that

$$
\left\|\operatorname{prox}_{f}(x)-\operatorname{prox}_{f}(y)\right\| \leq\left\|\operatorname{prox}_{f}(x)-z\right\|+\left\|\operatorname{prox}_{f}(y)-z\right\|<\|x-z\|+\|y-z\|<2 \epsilon .
$$

In particular, letting $y=\bar{x} \in S$ in (19), we have that

$$
\left\|\operatorname{prox}_{f}(x)-\bar{x}\right\|<\|x-\bar{x}\| \quad \forall x \in \mathbb{R}^{n} \backslash S,
$$

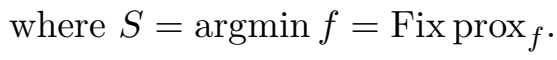


Example B.3 (non-averaged resolvent of quasiconvex function). The function $f(x):=1-$ $\exp \left(-\|x\|^{2}\right)$ for $x \in \mathbb{R}^{n}$ satisfies all the conditions in Lemma B.2. Its proximity operator has the derivative $\operatorname{prox}_{f}^{\prime}(A(x))=\left(A^{\prime}(x)\right)^{-1}$, where $A(x)=\left(1+2 \exp \left(-\|x\|^{2}\right)\right) x$, i.e. $A^{\prime}(x)=$ $\left(1+2 \exp \left(-\|x\|^{2}\right)\right)$ Id $-4 \exp \left(-\|x\|^{2}\right) x x^{\top}$. Since $\left\|\operatorname{prox}_{f}^{\prime}(A(x))\right\| \geq\|y\| /\left\|A^{\prime}(x) y\right\|$ for any $y \in$ $\mathbb{R}^{n} \backslash\{0\}$, we have with $x=e_{1}=(1,0, \ldots, 0)^{\top}=y$ that $\left\|\operatorname{prox}_{f}^{\prime}\left(A\left(e_{1}\right)\right)\right\|>1$, which is in contradiction to nonexpansiveness of averaged mappings, that have derivative bounded by 1 , if it exists.

Where paracontractions also occur are nonconvex feasibility problems, both consistent and inconsistent. As long as the fixed point set of the averaged projections operator consists of isolated points and the projectors are single-valued in a neighborhood of this fixed point, [25, Theorem 3.2] shows that these operators are paracontractions, whenever all assumptions of the theorem are met. Unfortunately, a statement on paracontractiveness, for the case that the fixed point set of the averaged projections operator does not consist of isolated points, is however not possible in general.

Furthermore, also nonconvex forward-backward operators appearing in structured optimization of nonconvex objective functions show the paracontractiveness property, see [25, Proposition 3.9], and these are not averaged in general, still the assumption that the fixed points are isolated is used.

\section{References}

[1] D. Butnariu and S. D. Flåm, "Strong convergence of expected-projection methods in hilbert spaces," Numerical Functional Analysis and Optimization, vol. 16, no. 5-6, pp. 601-636, 1995.

[2] A. Nedić, "Random algorithms for convex minimization problems," Mathematical Programming, vol. 129, no. 2, pp. 225-253, 2011.

[3] S. D. Flåm, "Successive averages of firmly nonexpansive mappings," Mathematics of Operations Research, vol. 20, no. 2, pp. 497-512, 1995.

[4] D. Butnariu, "The expected-projection method: Its behavior and applications to linear operator equations and convex optimization," Journal of Applied Analysis, vol. 1, no. 1, pp. 93-108, 1995.

[5] A. Nedić, "Random projection algorithms for convex set intersection problems," 49th IEEE Conference on Decision and Control, pp. 7655-7660, 2010.

[6] D. R. Luke, M. Teboulle, and N. H. Thao, "Necessary conditions for linear convergence of Picard iterations and application to alternating projections," arXiv, 2017.

[7] O. Kallenberg, Foundations of Modern Probability. Probability and Its Applications, New York: Springer, 1997.

[8] H. H. Bauschke and P. L. Combettes, Convex Analysis and Monotone Operator Theory in Hilbert Spaces. Berlin: Springer, 2011.

[9] W. R. Mann, "Mean value methods in iterations," Proc. Amer. Math. Soc., vol. 4, pp. 506510, 1953. 
[10] M. A. Krasnoselski, "Two remarks on the method of successive approximations," Math. Nauk. (N.S.), vol. 63, no. 1, pp. 123-127, 1955. (Russian).

[11] M. Edelstein, "A remark on a theorem of M. A. Krasnoselski," Amer. Math. Monthly, vol. 73, pp. 509-510, May 1966.

[12] L. Gubin, B. Polyak, and E. Raik, "The method of projections for finding the common point of convex sets," USSR Comput. Math. and Math. Phys., vol. 7, no. 6, pp. 1-24, 1967.

[13] J. B. Baillon, R. E. Bruck, and S. Reich, "On the asymptotic behavior of nonexpansive mappings and semigroups in Banach spaces," Houston J. Math., vol. 4, no. 1, pp. 1-9, 1978.

[14] M. Hairer, "Ergodic properties of Markov processes," Lecture Notes in Mathematics, vol. 1881, pp. 1-39, 2006.

[15] F. Deutsch, Best Approximation in Inner Product Spaces. New York: Springer, 2001.

[16] A. Y. Kruger, D. R. Luke, and N. H. Thao, "Set regularities and feasibility problems," Mathematical Programming, vol. 168, no. 1-2, pp. 279-311, 2018.

[17] J. Bolte, T. P. Nguyen, J. Peypouquet, and B. W. Suter, "From error bounds to the complexity of first-order descent methods for convex functions," Mathematical Programming, vol. 165, pp. 471-507, Oct 2017.

[18] H. Alt, Lineare Funktionalanalysis: Eine anwendungsorientierte Einführung (in German). Springer Lehrbuch, Berlin: Springer, 2002.

[19] V. Bogachev, Measure Theory, vol. I. Springer Berlin Heidelberg, 2007.

[20] M. Hairer, "Convergence of Markov processes," Lecture notes, University of Warwick, 2016.

[21] H. Bauer, Maß- und Integrationstheorie (in German). De Gruyter Lehrbuch, Berlin: W. de Gruyter, 1992.

[22] H. Robbins and D. Siegmund, "A convergence theorem for non negative almost supermartingales and some applications," in Optimizing Methods in Statistics (J. S. Rustagi, ed.), pp. 233 - 257, Academic Press, 1971.

[23] H. H. Bauschke and J. M. Borwein, "On projection algorithms for solving convex feasibility problems.," SIAM Rev., vol. 38, no. 3, pp. 367-426, 1996.

[24] K. J. Arrow and A. C. Enthoven, "Quasi-concave programming.," Econometrica, vol. 29, pp. 779-800, 1961.

[25] D. R. Luke, N. H. Thao, and M. K. Tam, "Quantitative convergence analysis of iterated expansive, set-valued mappings," Mathematics of Operations Research, pp. 1-33, 2018. 